\title{
High-Fidelity Reprogrammed Human IPSCs Have a High Efficacy of DNA Repair and Resemble hESCs in Their MYC Transcriptional Signature
}

\author{
Pratik K. Nagaria, ${ }^{1}$ Carine Robert, ${ }^{1}$ Tea Soon Park, ${ }^{2}$ Jeffrey S. Huo, ${ }^{2}$ \\ Elias T. Zambidis, ${ }^{2}$ and Feyruz V. Rassool ${ }^{1}$ \\ ${ }^{1}$ Department of Radiation Oncology, University of Maryland School of Medicine, Baltimore, MD 21201, USA \\ ${ }^{2}$ Institute for Cell Engineering and Sidney Kimmel Comprehensive Cancer Center, The Johns Hopkins University School of Medicine, \\ Baltimore, MD 21205, USA \\ Correspondence should be addressed to Feyruz V. Rassool; frassool@som.umaryland.edu
}

Received 18 March 2016; Revised 23 June 2016; Accepted 14 July 2016

Academic Editor: Silvia Brunelli

Copyright ( 2016 Pratik K. Nagaria et al. This is an open access article distributed under the Creative Commons Attribution License, which permits unrestricted use, distribution, and reproduction in any medium, provided the original work is properly cited.

\begin{abstract}
Human induced pluripotent stem cells (hiPSCs) are reprogrammed from adult or progenitor somatic cells and must make substantial adaptations to ensure genomic stability in order to become "embryonic stem cell- (ESC-) like." The DNA damage response (DDR) is critical for maintenance of such genomic integrity. Herein, we determined whether cell of origin and reprogramming method influence the DDR of hiPSCs. We demonstrate that hiPSCs derived from cord blood (CB) myeloid progenitors (i.e., CB-iPSC) via an efficient high-fidelity stromal-activated (sa) method closely resembled hESCs in DNA repair gene expression signature and irradiation-induced DDR, relative to hiPSCs generated from CB or fibroblasts via standard methods. Furthermore, sa-CB-iPSCs also more closely resembled hESCs in accuracy of nonhomologous end joining (NHEJ), DNA doublestrand break (DSB) repair, and C-MYC transcriptional signatures, relative to standard hiPSCs. Our data suggests that hiPSCs derived via more efficient reprogramming methods possess more hESC-like activated MYC signatures and DDR signaling. Thus, an authentic MYC molecular signature may serve as an important biomarker in characterizing the genomic integrity in hiPSCs.
\end{abstract}

\section{Introduction}

Although human induced pluripotent stem cells (hiPSCs) resemble hESCs in many respects [1,2], the therapeutic utility of hiPSCs is limited by low reprogramming efficiency [3-6] and poor genomic integrity [7-10]. A deeper understanding of the mechanisms that control these roadblocks will be vital for the use of hiPSCs in regenerative medicine. Reprogramming efficiency is controlled by intrinsic and extrinsic microenvironmental factors that are determined by the method employed [5]. Standard protocols often utilize inefficient and potentially mutagenic retroviral mediated transgene factor expression (e.g., OSKM: OCT4, SOX2, KLF4, and C-MYC, or OSNL, i.e., OCT4, SOX2, NANOG, and LIN28) [11]. More clinically useful nonviral, nonintegrating methods have also been widely employed (e.g., plasmids, microRNA), albeit with a significantly reduced reprogramming efficiency [11]. The choice of somatic donor and in vitro microenvironmental conditions also significantly influences reprogramming efficiency. For example, we previously demonstrated that bone marrow stromal cell (MSC) activation robustly activated MYC complex-regulated genes of pluripotency that subsequently facilitated high-quality reprogramming of human myeloid progenitors (MP) differentiated from $\mathrm{CD} 34^{+}$ hematopoietic stem-progenitor cells [12]. Activation of MYCregulated factors potentially enhanced the rate and efficiency of reprogramming [13]. MYC may also play a key role in regulating promoters and microRNAs associated with core pluripotency-associated genes [14, 15]. These findings implicate targets of the MYC network not only in playing a 
key role in controlling the efficiency of reprogramming, but also in maintaining stem cell pluripotency.

Efficacious DNA double-strand break (DSB) repair is a key element in maintenance of high genomic integrity [16, 17]. In mammalian cells, homologous recombination repair (HR) provides precise, error-free DSB repair by using a homologous sister chromatid as a template for repair [18]. In contrast, repair by nonhomologous end joining (NHEJ) joins DNA ends directly and is thus prone to errors [19]. In hESCs, repair of DSBs occurs mainly by HR [17]. We and others have reported a form of DSB end-joining repair in hESCs that is relatively error-free [17, 20, 21]. However, overall DNA repair properties in reprogrammed cells are more heterogeneous than hESCs $[22,23]$. For example, we previously demonstrated that hiPSCs derived from mesenchymal stem cells (MSCs) or fibroblasts were more deficient than hESCs in DSB end-joining capacity despite similarities in the precision of repair between them [20]. These studies suggest that efficient DSB repair properties confer an advantage in achieving completion of faithful reprogramming to an authentic hESClike state [24]. However, the mechanisms that control efficient DSB repair during reprogramming are unclear.

MYC, which can associate with the E-box elements in the promoters of several DSB repair genes and can amplify the cell's transcriptional program by binding to promoter and enhancer elements, represents a strong candidate for regulation of DSB repair in pluripotent cells $[25,26]$. Determining these mechanisms not only is critical in finding the most efficient way to derive iPSCs, but also can be applied to measures ensuring the safe clinical use of iPSCs with high genomic integrity. To address these questions, we evaluated previously reported human CB-derived sa-CBiPSCs generated with high efficiencies (1-4\% input cells) and compared them to CB- and fibroblast-derived hiPSCs derived via standard methods $(<0.001-0.5 \%$ input cells $)$ [27]. Our data reveal that in response to radiation-induced DNA damage, sa-CB-iPSCs possessed a DDR signature that more closely resembles that of hESCs. These sa-CB-iPSCs also possess lower baseline levels of endogenous DNA DSBs and a greater accuracy of DSB end-joining, compared to standard CB-iPSCs and fibroblast-iPSCs. Moreover, we show that CMYC may play an important role in facilitating a stringent and high-fidelity DSB response in hESCs and hiPSCs. Collectively, our data suggest that more efficient activation of MYC-associated DDR signaling during reprogramming or DSB damage may enhance the genomic integrity of hiPSCs and increase their ultimate clinical utility.

\section{Materials and Methods}

Ethics Statement (Human Embryonic Stem Cell Lines). All hESC lines used in this study were obtained commercially from the WiCell Research Institute (Wisconsin International Stem Cell Bank, WISCB). The use of all WISCB-donated hESC lines in these studies was approved by the Johns Hopkins Embryonic Stem Cell Research Oversight (JHUESCRO) Committee and the University of Maryland School of Medicine Embryonic Stem Cell Research Oversight Committee (UMSOM-ESCRO) and conforms strictly to standards of both institutions, including written informed consent. All experiments conducted in these studies also conformed to guidelines outlined for hESC and pluripotent stem cell research by the National Academy of Sciences and the National Institutes of Health (NIH).

2.1. Cell Culture. Pluripotent stem cells were routinely cultured on irradiated primary murine embryonic fibroblasts (MEF), derived from embryos of CF1 and DR4 F1 mice at embryonic days of 12.5 or 13.5 (P2/P3), or purchased from GlobalStem (Rockville, MD). Human pluripotent stem cell cultures were maintained in DMEM/F12 (Invitrogen) medium supplemented with 20\% Knockout Serum Replacement (KOSR; Gibco), 0.1 mM MEM nonessential amino acids (Gibco), $1 \mathrm{mM}$ L-glutamine (Gibco), $0.1 \mathrm{mM}$ $\beta$-mercaptoethanol (Sigma-Aldrich, St. Louis, MO), and $4 \mathrm{ng} / \mathrm{mL}$ FGF2 (R\&D Systems, Minneapolis, MN) at $37^{\circ} \mathrm{C}, 5 \%$ $\mathrm{CO}_{2}$, and $85 \%$ relative humidity. The medium was changed daily on hESCs and hiPSC cultures. For experiments, human pluripotent stem cells were first transitioned from $\mathrm{MEF}$ feeder layers onto a BD-Matrigel ${ }^{\mathrm{TM}}$ (BD Biosciences) matrix precoated plate and cultured in TTESR $^{\mathrm{TM}}{ }^{\mathrm{TM}}$ medium (Stem Cell Technologies, Vancouver, Canada). The mTESR1 growth media were replenished daily. Purified (>95\%) human CD34 ${ }^{+}$ $\mathrm{CB}$ progenitors (also referred to as "starting $\mathrm{CB}$ progenitors") from pooled donors were purchased from AllCells (Emeryville, $\mathrm{CA}$ ) and cultured in the hematopoietic growth medium (HPGM).

2.2. Generation of Episomal hiPSCs. Detailed methods for generation and characterization of hiPSC lines were previously described $[12,28]$. Details of hiPSC lines are provided in Table S1 in Supplementary Material available online at http://dx.doi.org/10.1155/2016/3826249. In brief, sa-CB-iPSC lines (CB6.2, 6.13, 19.11, and E12C1) were derived via nucleofection of stromal-activated CD $34^{+} \mathrm{MP}$ with 7 or 4 episomal factors (7F, SOKMNLT; SOX2, OCT4 (POU5F1), KLF4, cMYC, NANOG, LIN28, and SV40L T antigen; 4F, SOKM) using the AMAXA II Nucleofector device (Lonza). Standard episomal CB-iPSC lines were derived without stromal activation with either four (4F; SOKM) or seven episomal factors (7F) from either CB-derived CD $34^{+} \mathrm{MP}(4 \mathrm{~F}$ : E17C1, E20C2, and $\mathrm{E} 24 \mathrm{Cl}$ ) or $\mathrm{CB}$-derived unsorted mononuclear cells (7F: iCB9, iCB8, and iCB2.5) [29], kindly provided by Dr. Igor Slukvin (University of Wisconsin-Madison). Skin fibroblastderived hiPSC line iHUF3, derived with four retroviral factors (SOKM), was previously described (Byrne et al.) and kindly provided by Dr. Renee Reijo-Pera (Stanford University) [27]. Requests for hiPSC lines should be addressed to Elias T. Zambidis (ezambid1@jhmi.edu).

2.3. Gene Expression Microarrays. Details of the microarray analysis were described before [12]. Human HT-12 Expression BeadChip arrays (Illumina, San Diego, CA) were used for microarray hybridization to examine the global gene expression of hESC, hiPSC, and starting populations $\left(\mathrm{CD} 34^{+}\right.$progenitors and fibroblasts). The NIH Gene Expression Omnibus has issued the accession numbers GSE44425 (Figure 1, 


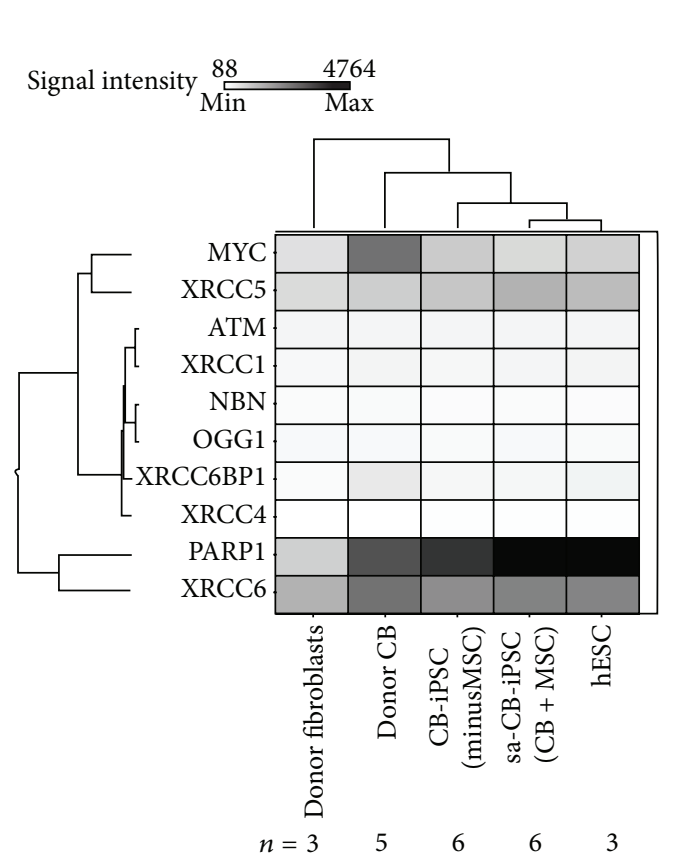

(i)

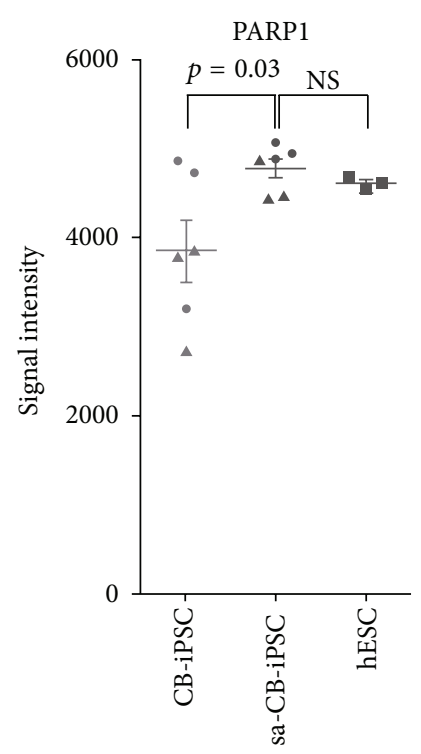

A 4F CB-iPSC

- 7F CB-iPSC

- hESC

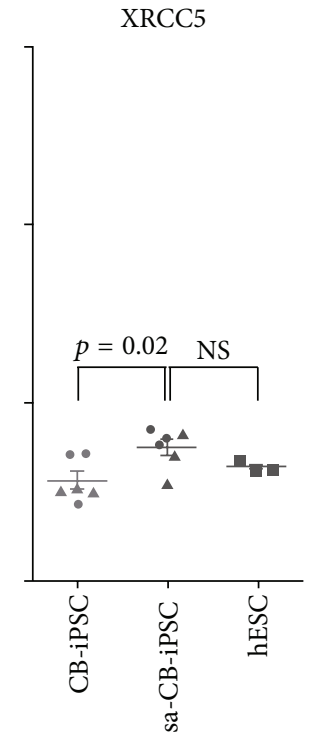

- $4 \mathrm{~F} \mathrm{CB}$-iPSC

- $7 \mathrm{~F}$ CB-iPSC

- hESC

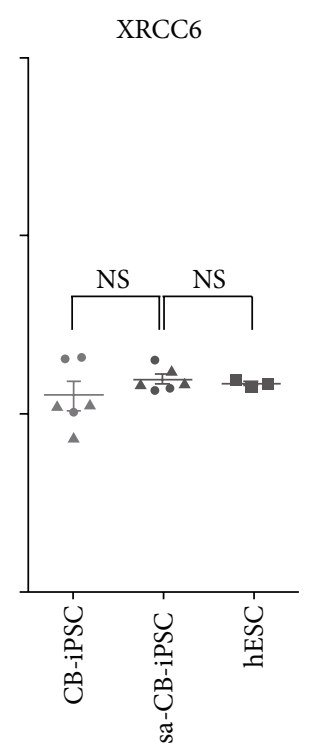

- $4 \mathrm{~F} \mathrm{CB}-\mathrm{iPSC}$

- $7 \mathrm{~F} \mathrm{CB}-\mathrm{iPSC}$

- hESC

(ii)

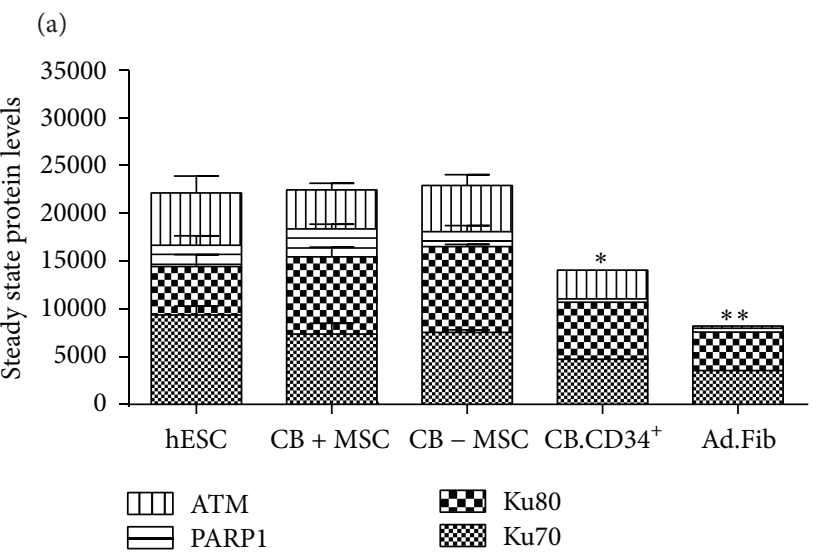

(ii)

(b)

FIGURE 1: CB progenitors and CB-derived iPSCs closely resemble hESCs DNA repair gene expression signature. Microarray gene expression of selected DNA repair genes. (a)(i) Shown are hierarchical clustering heatmaps of mRNA from donor fibroblasts, donor $\mathrm{CD} 34^{+} \mathrm{CB}$, and CB.iPSC derived with $(+\mathrm{MSC})$ and without $(-\mathrm{MSC})$ bone marrow stromal cell activation. hiPSC lines included sa-CBiPSC derived from stromal-activated CD34 $4^{+} \mathrm{MP}\left(n=6\right.$; E5C3, E12C5, and E17C6: 6.2, 6.13, and 19.11), standard CB-iPSC, lines derived from CD34 ${ }^{+}$MP without stromal activation $(n=3, \mathrm{E} 17 \mathrm{C} 1, \mathrm{E} 20 \mathrm{C} 2$, and E24C1), and standard CB-iPSC lines derived from CB unsorted mononuclear cells $(n=3$, iCB9, iCB8, and iCB2.5). hESC lines included $(n=3)$ H9, H7, and ES03. Signal intensities are from averaged independent biological replicate microarray samples ( $n$ as indicated). Expression array data depicts normalized values of the mean transcript levels for a subset of DDR genes in each group of the indicated cell lines. (a)(ii) Dot plots represent the normalized values of the signal intensities for PARP1/XRCC5/XRCC6 with corresponding $p$ values between categories indicated in the array data in (a)(i) ( $n$ as indicated in (a)(i)). Paired tests with significance $p<0.05$ $(*)$ or without significance (NS; $p>0.05)$ with values of control hESC are indicated $(\boldsymbol{\Delta}=4 \mathrm{~F} \mathrm{CB} . i \mathrm{PSC} ; \boldsymbol{\bullet}=7 \mathrm{~F}$ CB.iPSC). (b)(i) Representative Western blot from the whole cell lysates of hESCs (H9 and ES03), CB (CD34 ${ }^{+}$), two independent sa-CB-iPSCs (6.2 and E12C1), and adult fibroblasts (Ad.Fib) showing the steady state levels of PARP1 and Ku80 and ATM. $\beta$-Actin was used as the loading control. (b)(ii) Graphical representation of Western blots by ImageJ quantified-densitometry analysis normalized to $\beta$-actin $(n=3)$ in hESC (H9, ES03, and H7), sa-CB-iPSC (CB6.2, CB6.13, and CB19.11), standard CB mononuclear CB-iPSC (iCB9, iCB8, and iCB2.5), CB (CD34 ${ }^{+}$), and adult fibroblasts (Ad.Fib). Results are representative of the mean of two independent experiments of each set $\pm \mathrm{SEM},{ }^{*} p<0.05$ and ${ }^{* *} p<0.01$, based on 2-way ANOVA (multiple comparisons test) on combined expression of genes. 
Figure 5(c)) for the deposited microarray data related to the above manuscript.

2.4. DNA Damage, Apoptosis, and MYC Inhibition Studies. For irradiation (IR) studies, pluripotent stem cells were exposed to X-ray radiation using a Pantak HF320 X-Ray machine $(250 \mathrm{kV}$ peak, $13 \mathrm{~mA}$; half-value layer, $1.65 \mathrm{~mm}$ copper) at a dose rate of $2.4 \mathrm{~Gy} / \mathrm{min}$. For experiments involving MYC inhibitor (10058-F4, Sigma-Aldrich, St. Louis, MO), the cells were treated with either the control solvent (DMSO) or the drug at dose of $50 \mu \mathrm{M}$ for $24 \mathrm{~h}$ before X-ray IR. Following $24 \mathrm{~h}$ treatment, the medium was replaced before exposure to IR. For knockdown studies, siMYC (ON-TARGETplus ${ }^{\mathrm{TM}}$, Dharmacon, Thermo Fisher Scientific) was utilized. The cells were transfected with siMYC $(2 \mu \mathrm{g})$ using Lipofectamine ${ }^{\mathrm{TM}}$ 2000 (Life Technologies), $48 \mathrm{~h}$ before exposure to IR.

2.5. Whole Cell Extracts and Nuclear Extracts. Whole cell extracts were prepared with lysis buffer $(25 \mathrm{mM}$ Tris- $\mathrm{HCl}$ (pH 7.5), $333 \mathrm{mM} \mathrm{KCl,} 1.3 \mathrm{mM}$ EDTA, and $4 \mathrm{mM} \mathrm{DTT}$ ) with protease inhibitor cocktail (Roche, Branchburg, NJ) and phosphatase inhibitors cocktail (Sigma-Aldrich). Nuclear extracts were prepared using the CelLytic Nuclear Extraction Kit (NEXTRACT ${ }^{\mathrm{TM}}$, Sigma-Aldrich) without the use of any detergents. The nuclear extracts used for the DNA repair assay were dialyzed against the E-buffer $(20 \mathrm{mM}$ Tris- $\mathrm{HCl}$ (pH 8.0), 20\% glycerol, 0.1 M K(OAc), $0.5 \mathrm{mM}$ EDTA, and $1 \mathrm{mM}$ DTT).

2.6. Immunoblotting Analysis. $20 \mu \mathrm{g}$ of proteins was separated by electrophoresis through either $4-10 \%$ or $4-15 \%$ polyacrylamide gradient gels (Mini-PROTEAN TGX) (BioRad Laboratories, Hercules, CA) and then transferred to PVDF membranes (Thermo Fisher Scientific, Waltham, MA). After blocking, membranes were probed with primary antibodies mouse Ku70 (1: 500, E-5, SC17789, Santa Cruz Biotech (SCB), Dallas, TX), Ku80 (Calbiochem, EMD Millipore NA54), PARP1 (1:2000, CS \# 9532, Cell Signaling, Beverly, MA), p53 (1:1000, CS \# 9282), pATM (1:1000, Millipore, Billerica, MA), $\gamma \mathrm{H} 2 \mathrm{AX}$ (Millipore, Clone JBW301, 05-636), $\beta$-actin (1:5000, Sigma-Aldrich), and $\beta$-tubulin (CS \# 2128) as loading controls. After probing with adequate secondary antibodies (anti-mouse IgG-CS and anti-rabbit, BioLegend, San Diego, CA), proteins expression was detected using enhanced chemiluminescence (ECL; $100 \mathrm{mM}$ Tris- $\mathrm{HCl}(\mathrm{pH}$ 8.5), luminol, coumaric acid, and hydrogen peroxide).

2.7. In Vitro NHEJ Assays (Plasmid Reactivation: PUC18 and EJ5-ISce1). We used the DNA repair fidelity assay (PUC18-based) as described before [30]. For the assay, $2 \mu \mathrm{g}$ of EcoRI linearized PUC18 was incubated with $4 \mu \mathrm{g}$ of nuclear extract. Reactions (in $20 \mu \mathrm{L}$ volume) were carried out in ligation buffer $(50 \mathrm{mM}$ triethanolamine- $\mathrm{HCl}(\mathrm{pH} 7.5)$, $60 \mathrm{mM}$ KOAc, $50 \mu \mathrm{M}$ deoxynucleotide triphosphates, $2 \mathrm{mM}$ ATP, $1 \mathrm{mM}$ DTT, and $100 \mu \mathrm{g} / \mathrm{mL}$ BSA). The mixture was incubated for $16 \mathrm{~h}$ at $18^{\circ} \mathrm{C}$. Following the incubation, $10 \mathrm{ng}$ of purified plasmid DNA was used to transfect Escherichia coli strain DH5 $\alpha$. Transformed cells were plated on LB agar plates, including $100 \mu \mathrm{g} / \mathrm{mL}$ carbenicillin, $20 \mathrm{mg} / \mathrm{mL}$ X-gal, and $200 \mathrm{mg} / \mathrm{mL}$ isopropyl-1-thio- $\beta$-d-galactopyranoside. To allow for spontaneous rejoining/incomplete EcoRI cutting, assay controls were conducted without nuclear extract. In addition, to correct for bacterial plating numbers and determine whether nuclease activity was affecting plasmid efficacy, cells were plated on Luria-Bertani agar without carbenicillin.

For the EJ5-Iscel assay, we used a protocol adapted from the one designed by Bennardo and colleagues but modified for in vitro plasmid reactivation analysis [31]. Briefly, the pimEJ5GFP reporter plasmid (Addgene Plasmid 44026) [31] was enzymatically linearized with I-Scel (New England Biolabs (NEB), Ipswich, MA) at $37^{\circ} \mathrm{C}$ overnight. Linearized plasmid was dephosphorylated using Shrimp Alkaline phosphatase (SAP) (NEB), and column $500 \mathrm{ng}$ DNA was incubated with dialyzed nuclear extracts $(2 \mu \mathrm{g})$ of respective cell lines, and ligation reactions were performed in ligation buffer (10x T4 ligase buffer, $2 \mathrm{mM}$ ATP, and $50 \mu \mathrm{M}$ deoxynucleotide triphosphates). Following in vitro ligation, the plasmid DNA was column-purified and GFP genes were PCR-amplified using the primers p1 (Fwd) $5^{\prime}$-CTGCTAACCATGTTCATGCC-3' and p2 (Rev) $5^{\prime}$-AAGTCGTGCTGCTTCATGTG-3', as described by Bennardo et al. [31]. Following the PCR, we redigested plasmid with $I-S c e 1$ to differentiate between NHEJ repair that was completed with $I-S c e 1$ restoration $(\mathrm{S}+)$ and repair completed with loss of I-Scel site (i.e., "S-" with deletions). Undigested and digested PCR products were fractionated on 2\% agarose and visualized with the GelStar ${ }^{\mathrm{TM}}$ Nucleic Acid Stain (Lonza). S-fragment was excised from the gel and cloned into PCR2.1 ${ }^{\circledR}$ TOPO (Life Technologies). Cloned products were transformed into OneShot ${ }^{\circledR}$ TOP10 chemically competent cells (Life Technologies) and plated on LB plates with kanamycin resistance. DNA from 5 colonies from each experiment was sequenced using the M13 primers. A total of 15 colonies were analyzed from three independent experiments, and TOPO plasmids were sequenced at the UMB TGL/Biopolymer core facility.

\section{Results}

3.1. CB Progenitors and CB-Derived iPSCs Closely Resemble hESCs in DNA Repair Gene Expression Signature. Previous studies indicated that progenitor donor cells were more amenable than differentiated cells in reprogramming to a pluripotent state $[32,33]$. We performed microarray-based analysis to determine the DDR gene expression profile of hiPSCs (Table S1) derived via different methods (Figure 1(a)(i)). We found that donor $\mathrm{CD} 34^{+} \mathrm{CB}$ progenitors cluster more closely with hESCs than adult fibroblasts (Ad.Fib) donors in baseline expression of DNA repair genes, including poly (ADP-ribose) polymerase 1-PARP1 (involved in single-strand break repair and DSB repair), XRCC5 (a.k.a. Ku80), and XRCC6 (a.k.a. Ku70) (involved in NHEJ DSB repair). Of note, expression of MYC and XRCC6 in CB progenitors was even higher than that for hESCs (Figure 1(a)(i)) [20]. Additionally, PARP1 and XRCC5 were expressed at higher baseline levels in sa-CB-iPSCs than in standard CB-iPSC lines (Figure 1(a)(ii)). 
To determine whether the levels of expression of these repair gene transcripts translated into functional differences in protein levels, we performed immunoblot analyses on hiPSCs from these representative categories. Although steady state protein levels of ATM, Ku80, and PARP1 in sa-CBiPSCs were similar to standard CB-iPSCs and hESCs, donor $\mathrm{CD}_{3} 4^{+} \mathrm{CB}$ progenitor baseline expression of these DNA repair proteins more closely resembled hESCs $\left({ }^{*} p<0.05\right)$, compared to Ad.Fib ( ${ }^{* *} p<0.01$ difference) (Figures 1(b)(i) and 1(b)(ii)). These results suggested that CD34 ${ }^{+}$ CB progenitors may already possess hESC-like expression of DDR pathway components, even prior to initiation of reprogramming.

\subsection{Sa-CB-iPSCs Resemble hESCs in Their DDR Response to} Radiation. Irradiation (IR) elicits several posttranslational modifications of the components of DDR pathway. Irradiated hESCs and hiPSCs rapidly activate the ataxia telangiectasia and Rad3-related (ATR) and ataxia telangiectasia mutated (ATM) kinase-dependent DDR signaling [34], phosphorylating targets, such as p53 and $\operatorname{H} 2 \mathrm{AX}[17,35]$. While ATR responds mainly to single strand breaks (SSBs) and stalled replication forks, ATM is activated in response to DSBs. Moreover, ATM deficiency confers hypersensitivity to IR [36].

To determine the efficacy of DDR, representative CBderived hiPSCs (i.e., sa-CB-iPSC (CB6.2), standard CBderived hiPSC (iCB9), and fibroblast-derived (iHUF3)) [34] were treated with IR (2 Gy) and compared with IR-treated hESCs (i.e., H9 and ESO3). To examine the DSB response in IR-treated hiPSCs, we performed immunoblotting for phosphorylation of H2AX on Ser139 ( $\gamma \mathrm{H} 2 \mathrm{AX})$, which functions to assemble DSB repair factors [35]. In hESCs (H9 and ES03) and sa-CB-iPSC (CB6.2), $\gamma \mathrm{H} 2 \mathrm{AX}$ expression was evident at $4 \mathrm{~h}$ after IR (Figures 2(a) and 2(c)), indicating activation of a DSB response. All tested hiPSCs exhibited kinetics of H2AX phosphorylation similar to hESCs (Figures 2(a)-2(c)). Interestingly, hESCs and hiPSCs did not differ significantly in the expression levels of total ATM protein (Figures 2(a) and 2(b)). Notably, hESCs and CB-derived hiPSCs, including sa-CB-iPSC (CB6.2) and standard CB-iPSC (iCB9), demonstrated activation of ATM via phosphorylation at Ser1981 up to $4 \mathrm{~h}$ following IR (Figures 2(a)(i), 2(a)(ii), 2(b), and 2(d)). Interestingly, in comparison to hESCs and CB-iPSCs, fibroblast-derived iHuF3 cells exhibited less robust phosphorylation of ATM following exposure to $2 \operatorname{Gy} \operatorname{IR}\left({ }^{*} p<\right.$ 0.05 ) (Figures 2(a), 2(b), and 2(d)).

We next examined the activity of another ATM target, the tumor suppressor p53, whose expression is stabilized upon DNA damage, thus activating the DNA binding function of p53. Posttranslational modification of p53 via phosphorylation at Ser15 has been proposed to be an important mechanism by which p53 is stabilized and its functions are regulated [37]. However, phosphorylation is not an absolute necessity for DNA damage-induced stabilization of p53 [37]. Our results show that P53 activation, measured by monitoring total p53 protein and phosphorylation at Ser15, occurred with similar kinetics in all the hiPSCs and hESCs, with levels increasing between 0 and $4 \mathrm{~h}$ after IR (Figures 3(a)-3(c)).
Moreover, standard hiPSC lines (e.g., iCB9 and iHuF3) consistently displayed higher baseline levels of total p53 protein in untreated controls, in comparison to hESC (H9), ESO3, and sa-CB-iPSC (CB6.2) (Figures 3(a)-3(d)). In our observation, activation of p53 in cells following IR is mostly contributed by the stabilization of total p53 protein, as the relative changes in levels of phosphorylated protein were insignificant when its expression is normalized to total p53 (except for CB6.2 (2 h), $p<0.05$ ) (Figure S2).

We next investigated apoptotic responses of hiPSC cell lines to IR-induced damage. All pluripotent stem cells have been reported to exhibit hypersensitivity to radiation, with substantial cell death observed within $24 \mathrm{~h}$ after exposure to a low dose of ionizing radiation (1-2 Gy IR) [17, 20, 34]. We therefore reasoned that cells with higher levels of cytotoxic DSBs may induce apoptosis to avoid genotoxic stress. Using PARP1 cleavage as an apoptotic marker, IRexposed cells were examined by immunoblotting. Notably, there were only subtle differences observed in the kinetics of PARP1 cleavage among hESCs and all hiPSCs. PARP1 was observed predominantly in the cleaved form $4 \mathrm{~h}$ after IR in all examined cell lines (Figures 3(a), 3(b), and 3(d)). These results indicated that despite the subtle differences in levels of DNA damage, reprogramming renders all hiPSCs equally hypersensitive to ionizing radiation-induced apoptosis.

3.3. Sa-CB-iPSCs More Closely Resemble hESCs in Nonhomologous End Joining (NHEJ) Repair. Differences in baseline levels of DNA damage markers between hiPSCs noted above may also be accounted for by differences in DSB repair [17]. For example, increased DSB formation could result from decreased efficiency of repair, which can lead to increased error-prone repair or misrepair. Thus, we next determined whether the CB.iPSCs derived with the same factors but using distinct episomal reprogramming methods demonstrated different DSB repair efficiencies. We employed an established end-joining plasmid-reactivation repair assay and observed that hESC H9 and sa-CB-iPSC CB6.2 displayed the lowest percentage of misrepair (approximately 3\%). In contrast, standard hiPSCs iHuF3 and iCB9 possessed a significantly higher percentage of misrepair (approximately $8-12 \%$; ${ }^{*} p<$ 0.05), when either compatible DSB ends or noncompatible DSB ends (which require additional processing steps in end joining) were used (Figures 4(a)(i) and 4(a)(ii)). To further confirm these results, we utilized an additional modified endjoining assay designed by Gunn and Stark [38] that measures DSB repair junctions representing repair of complementary or noncomplementary ends (Figure S1). We incubated ISce1-linearized pimEJ5GFP plasmid with nuclear extracts of pluripotent cell lines for measurement of in vitro plasmid reactivation (Figure 4(b)(i)), and the $I$-Scel resistant fraction ("S-" products) was further analyzed for quantification and characterization of DNA deletions (Figure 4(b)(ii)). Sequencing of approximately 10-15 "S-" DNA clones recovered from end-joining experiments using $\mathrm{H} 9$ and CB6.2 extracts indicated that deletions in the DSB junctions were mainly in I-Scel overhangs and were restricted to 1-5 nucleotides (nt) $(33 \%$ and $54 \%$, resp.). In contrast, only 1 out of $11(9 \%)$ 


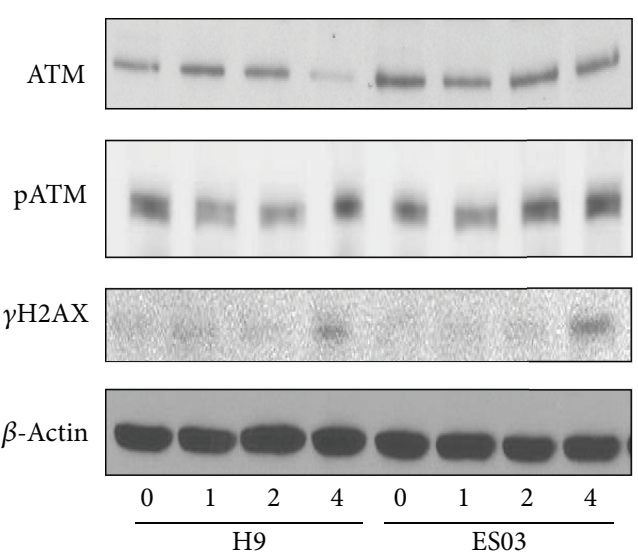

(i)

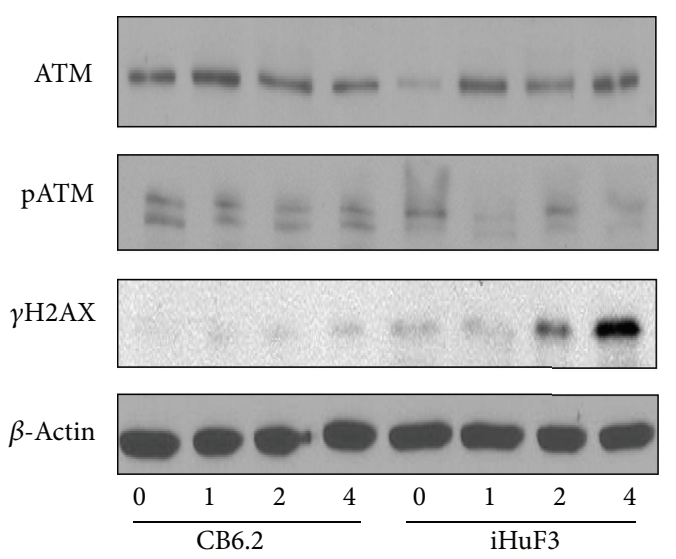

(ii)

(a)

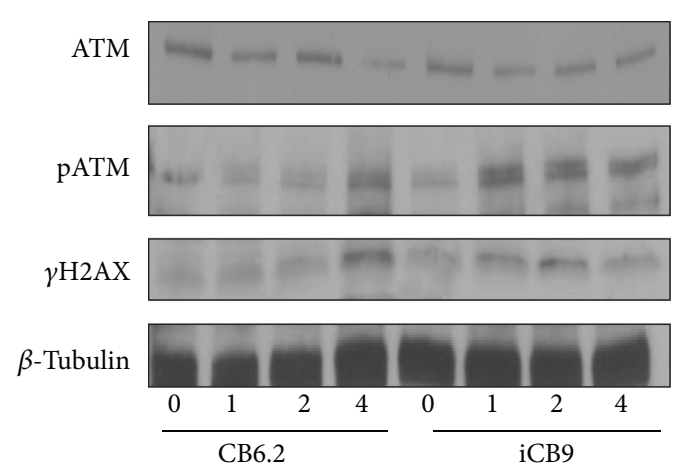

(b)

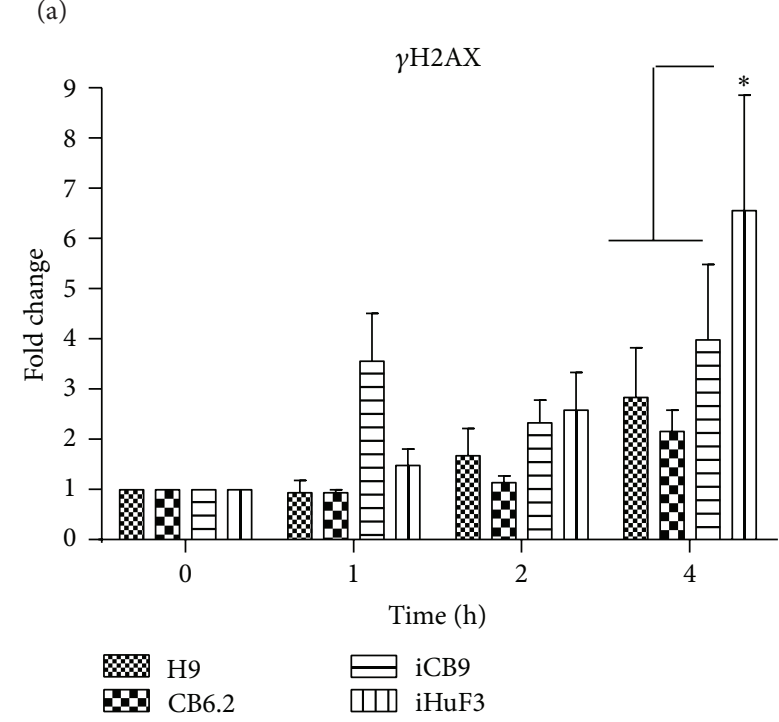

(c)

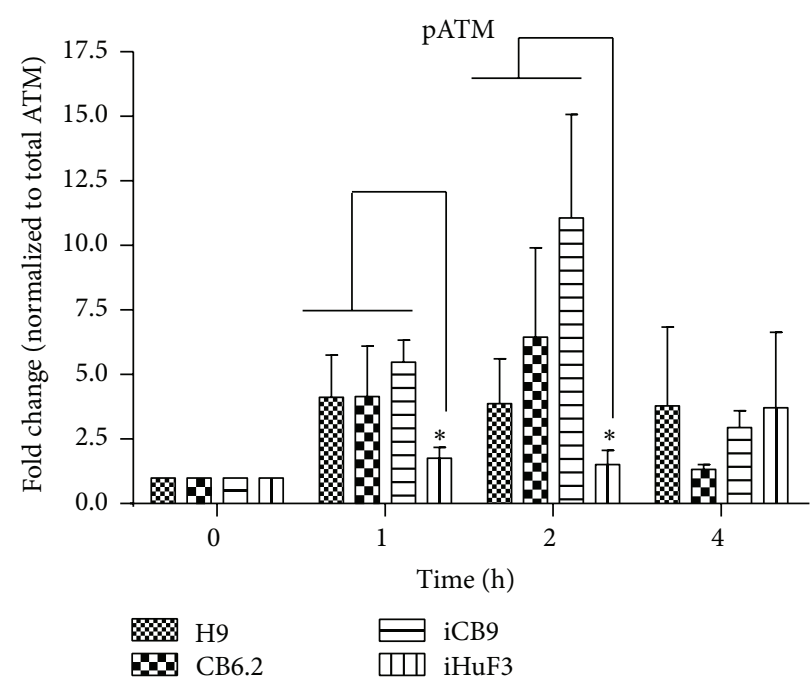

(d)

Figure 2: sa-CB-iPSCs closely resemble hESCs in DSB damage response to radiation. ((a)(i), (a)(ii), and (b)) Representative Western blot analysis depicting the expression of phosphorylated ATM (pATM) and H2AX ( $\gamma \mathrm{H} 2 \mathrm{AX})$ in cell lysates from H9, ES03, CB6.2, iCB9, and iHuF3 at time 0 and at $1 \mathrm{~h}, 2 \mathrm{~h}$, and $4 \mathrm{~h}$ after IR. $\beta$-Actin and $\beta$-tubulin were used as loading controls. Cells were exposed to IR (X-ray; 2 Gy) recovered at the indicated time points and immunoblotting was performed to analyze the kinetics of DDR protein expression. ((c) and (d)) Densitometry analysis of the Western blots for (c) $\gamma \mathrm{H} 2 \mathrm{AX}$ and (d) pATM (normalized to total ATM), using ImageJ software. Statistical significance of the data was determined using 2-way ANOVA analysis with Bonferroni posttests to compare the replicates (three independent experiments). $\gamma \mathrm{H} 2 \mathrm{AX}$ expression in iHuF3 is significantly different at $4 \mathrm{~h}$ compared to the following (versus $\mathrm{H} 9$ and $\mathrm{CB} 6.2,{ }^{*} p<0.05$ ). pATM expression in iHuF3 is significantly different at $1 \mathrm{~h}$ and $2 \mathrm{~h}$, compared to all other cell lines $\left({ }^{*} \mathrm{p}<0.05\right)$. 


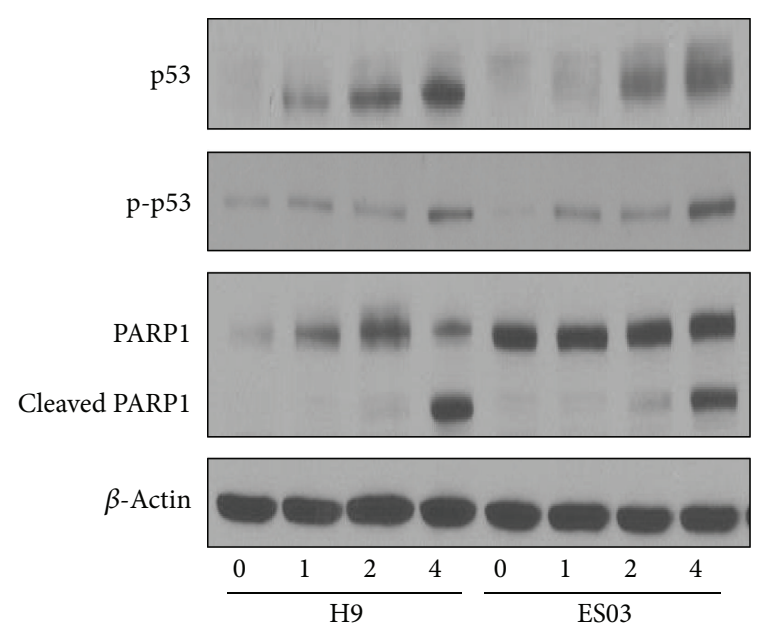

(i)

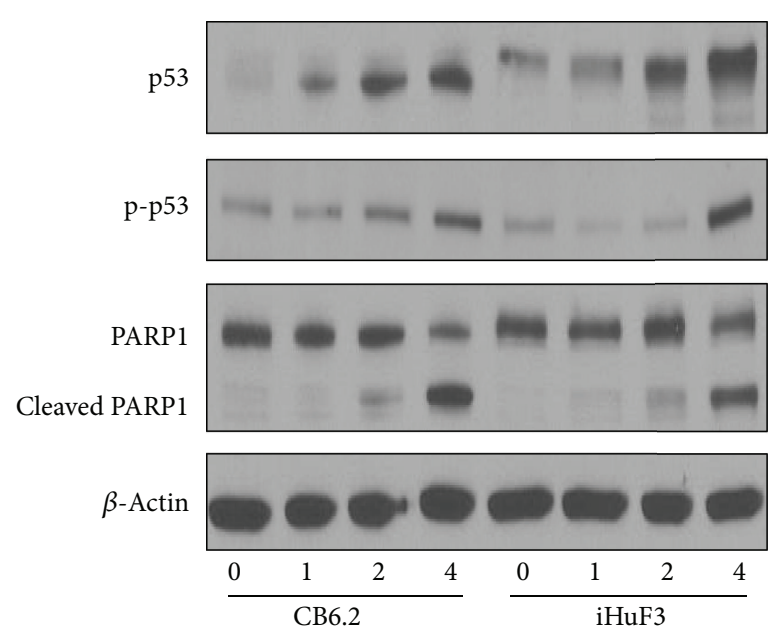

(ii)

(a)

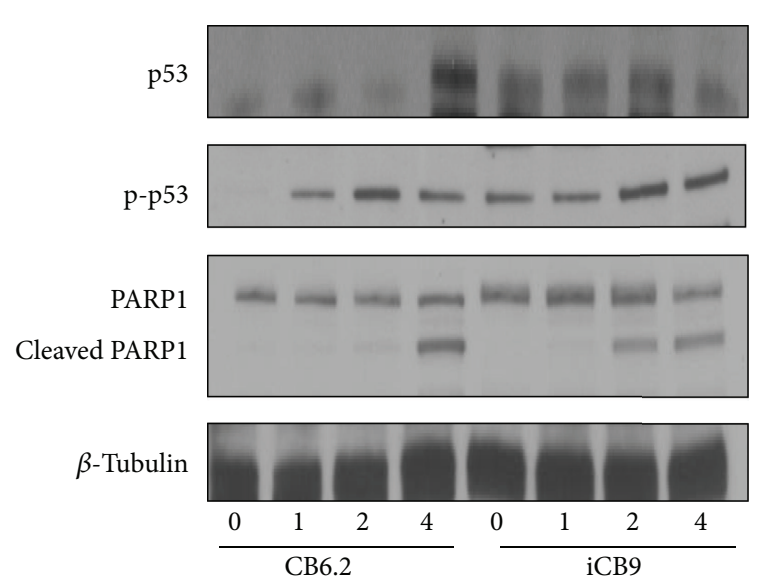

(b)

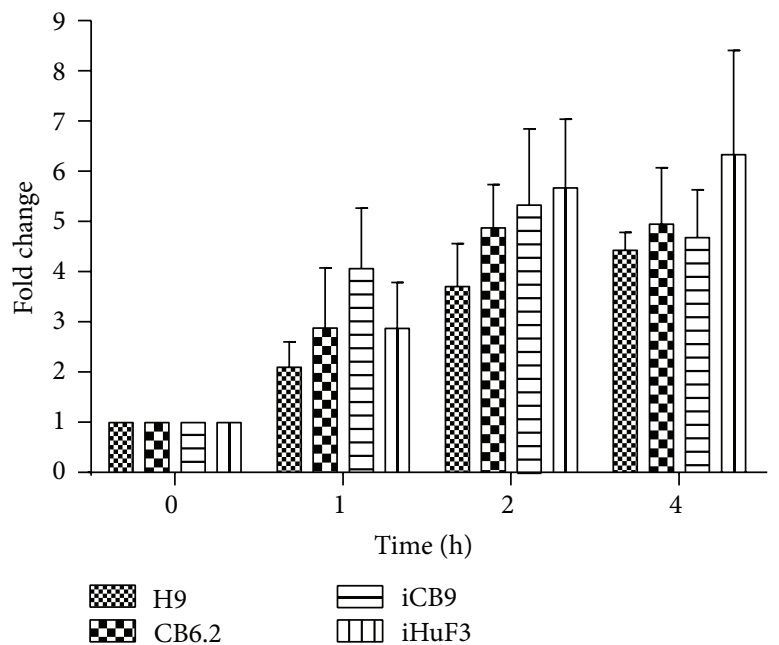

(d)

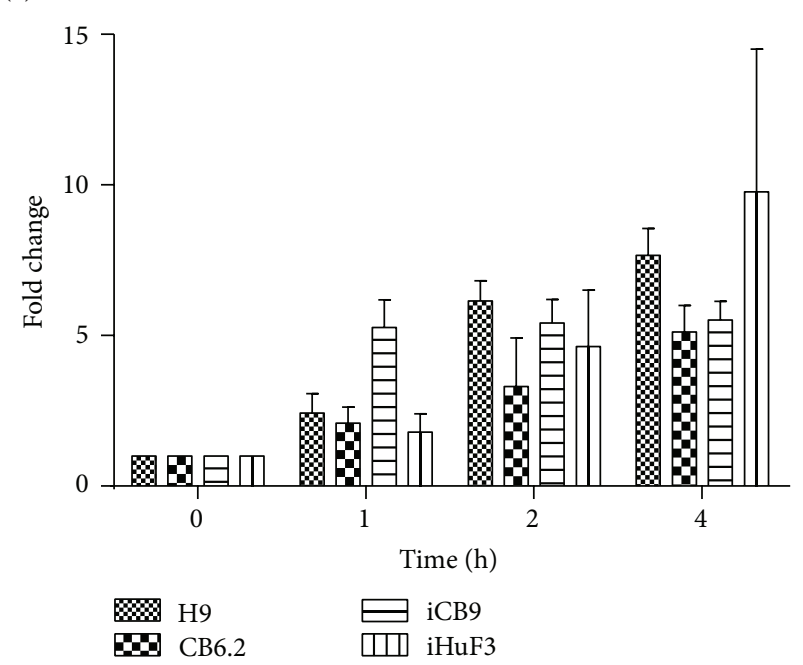

(c)

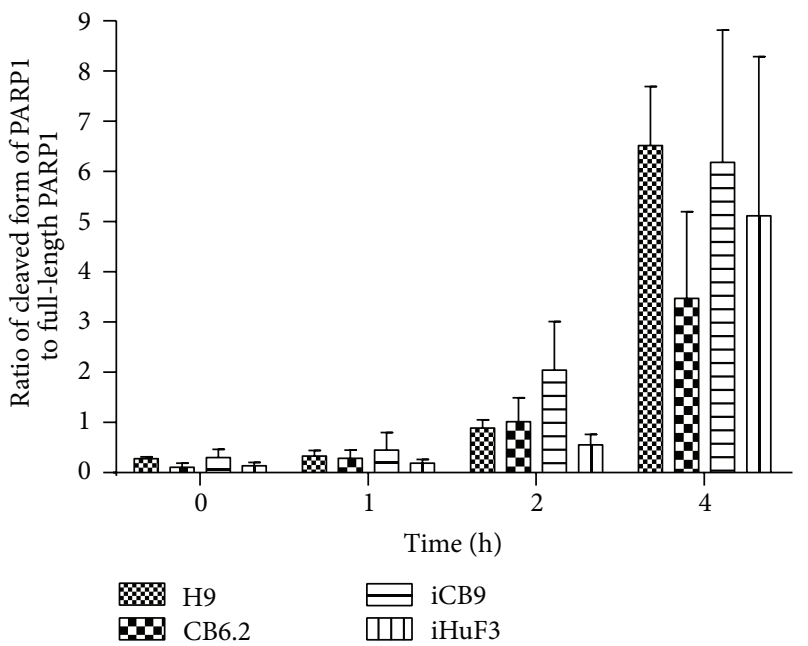

(e)

FIGURE 3: hESCs and hiPSCs have similar kinetics of apoptotic response to radiation exposure. ((a)(i), (a)(ii), and (b)) Representative Western blot analysis depicting the expression of p53 and PARP1 (full-length: $116 \mathrm{kDa}$; cleaved form: $89 \mathrm{kDa}$ ) in cell lysates from H9, ES03, CB6.2, iCB9, and iHuF3 at time $0 \mathrm{~h}$ and at $1 \mathrm{~h}, 2 \mathrm{~h}$, and $4 \mathrm{~h}$ after IR. (c-e) Densitometry analysis of the western blots for measurement of (c) total p53, (d) phosphorylated $\mathrm{p}^{\text {Ser15 }}$ (p-p53), and (e) PARP1 cleavage, using ImageJ software. Statistical significance of the data was determined using 2-way ANOVA with Bonferroni posttests to compare the replicates (three independent experiments). 


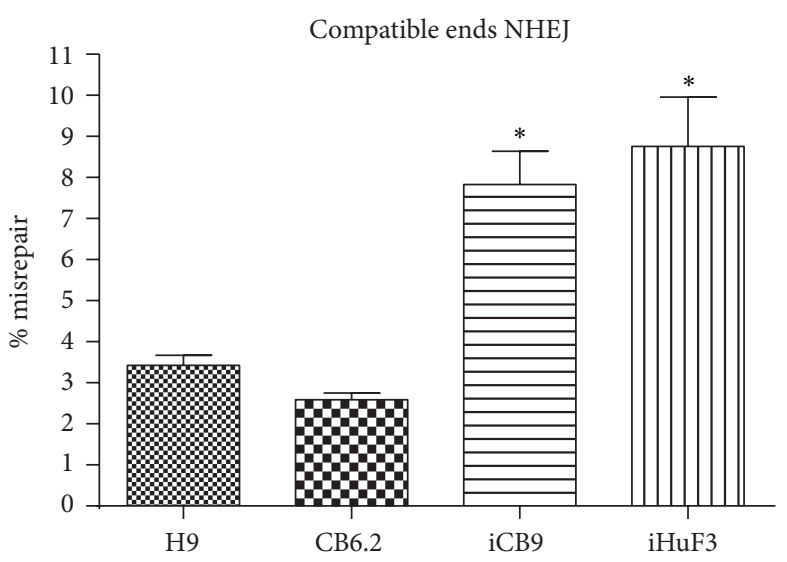

(i)

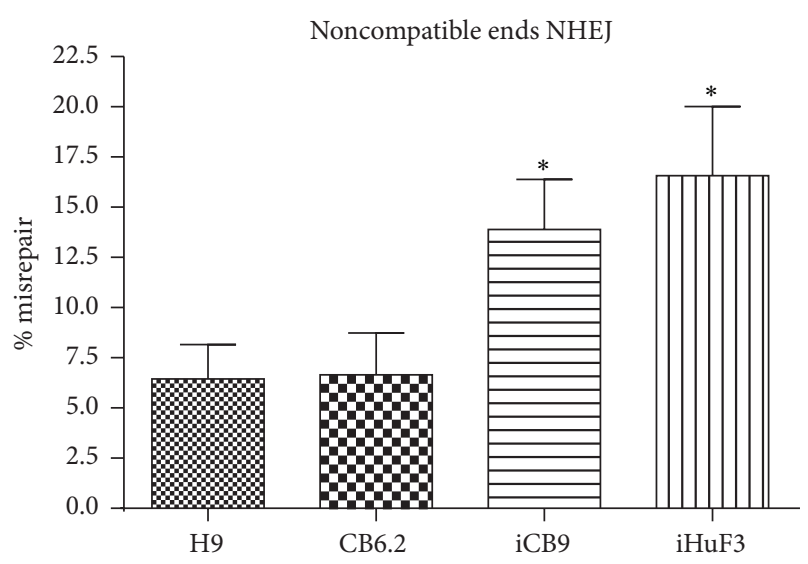

(ii)

(a)

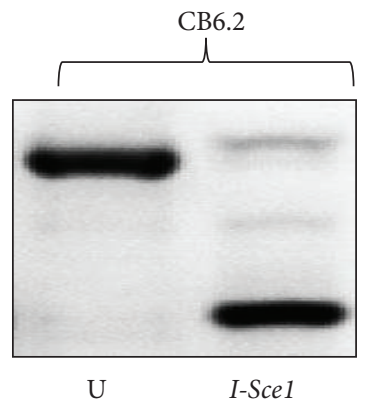

(i)

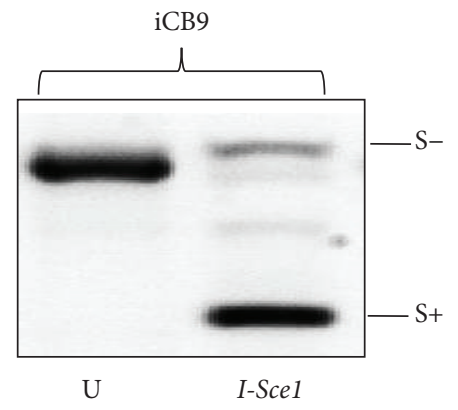

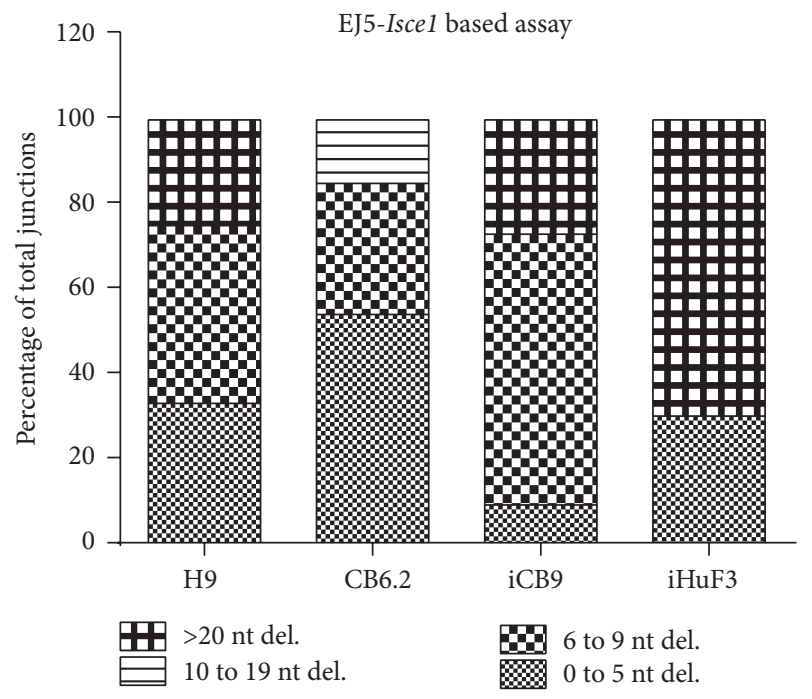

(ii)

(b)

FIGURE 4: sa-CB-iPSC closely resembled hESC showing greater accuracy of nonhomologous end joining (NHEJ) repair. ((a)(i) and (a)(ii)) Analysis of repair products indicating percentage of misrepair in the in vitro PUC18-based end-joining assay. The misrepair \% is calculated by dividing the total \# of white colonies by total \# of colonies, that is, blue + white, recovered from transformation of the repair products. (a)(i) demonstrates the \% misrepair when the dialyzed nuclear lysates from respective cell lines are incubated with PUC18 linearized using EcoR1, giving compatible DNA ends; and (a)(ii) demonstrates the \% misrepair when the dialyzed nuclear lysates from respective cell lines are incubated with PUC18 linearized using two restriction endonucleases (Kpnl/SacI), giving noncompatible DNA ends. Statistical significance of the data was determined using one-way ANOVA with Bonferroni posttests to compare all pairs of columns (cell lines). The data is significantly different for $\mathrm{H} 9$ or CB6.2 versus iCB9 or iHuF3 $\left({ }^{*} p<0.05\right)$. (b)(i) Shown is a representative gel image of the PCR products from CB6.2 and iCB9 that are redigested with $I-S c e 1$ or left uncut (U). All the S+ products on the gel represent correct repair that restores the I-Scel site in the plasmid. (S-) products represent the I-Sce1 resistant repair products, which were cloned into TOP10 competent cells. (b)(ii) The clones, each representing different repair products, were analyzed by sequencing across I-Scel junction. Data represents $\sim 10-15$ clones analyzed in H9, CB6.2, iCB9, and iHuF3. The data is significantly different for iCB9 versus H9 and CB6.2 (0-5 nt/6-9 nt deletions) or iHuF3 versus H9, CB6.2, and iCB9 ( $>20$ nt deletions) $\left({ }^{*} p<0.05\right)$.

junctions that were recovered from iCB9 extracts showed 15 nt deletions $\left({ }^{*} p<0.05\right.$, compared to H9, CB6.2), $64 \%$ of the junctions had 6-9 nt deletions, and $\sim 27 \%$ of the junctions had $>20$ nt deletions. Strikingly, we observed that $70 \%$ of junctions ( 7 out of 10) recovered from iHuF3 had deletions $>20$ nt $\left({ }^{*} p<0.05\right.$, compared to H9, CB6.2, and iCB9) (Figure 4(b)(ii)). This confirms that DNA end joining in saCB-iPSC CB6.2 more closely resembles that of hESCs and is less error-prone, compared to end-joining in the fibroblastderived standard hiPSCs.

3.4. C-MYC Maintains the DDR and NHEJ in hESCs and Is Required for Less Error-Prone Repair in sa-CB-iPSCs. MYC modules, along with Core and Polycomb group genes, represent key gene circuits that contribute to the ES cell expression signature [39]. C-MYC depletion from the reprogramming 


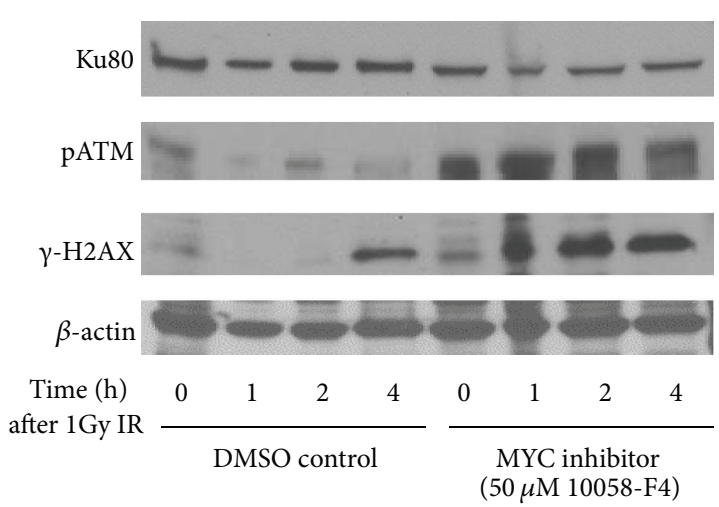

(i)

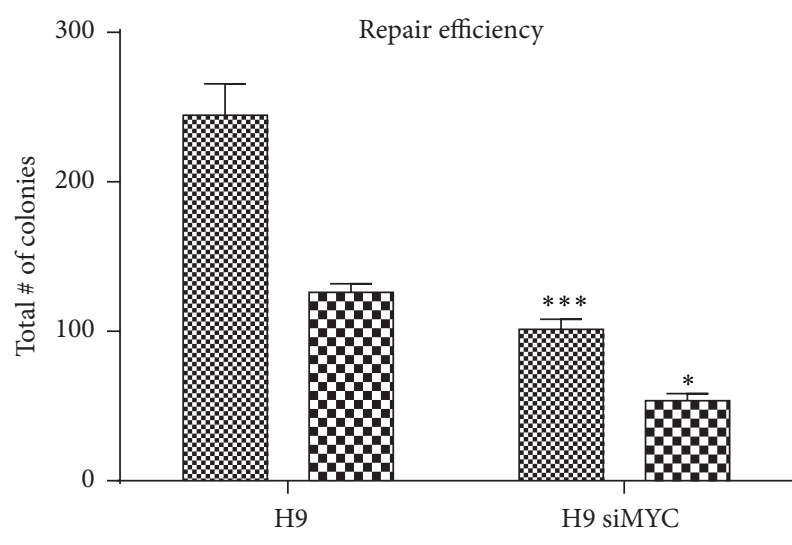

18. Compatible ends

0 Noncompatible ends

(i)

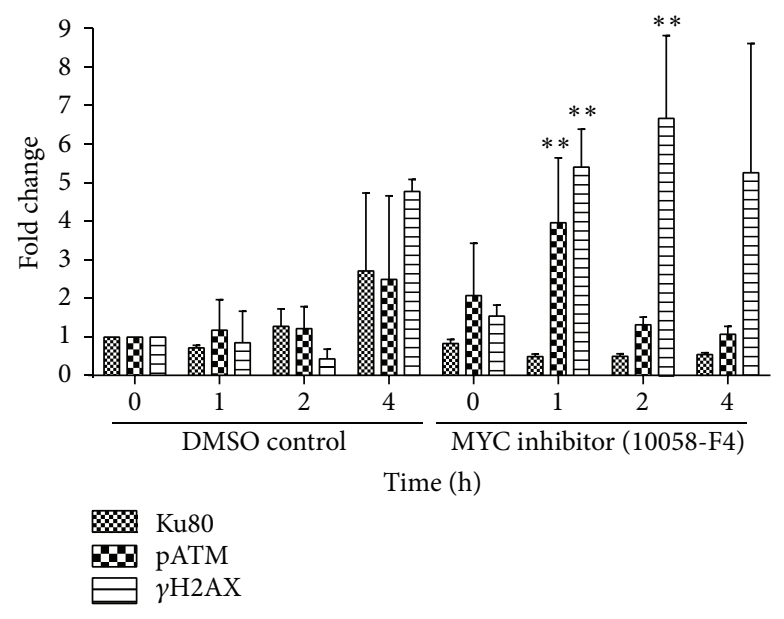

(ii)

(a)

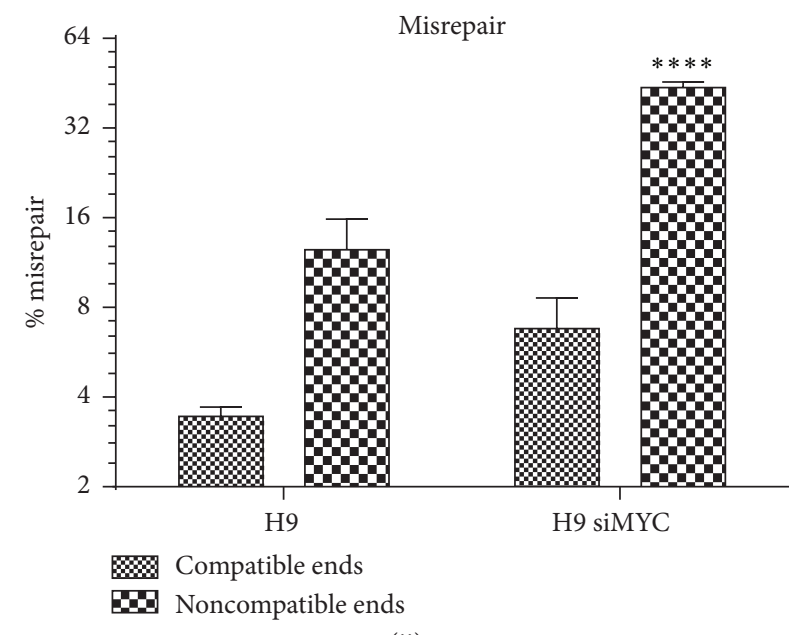

(ii)

(b)

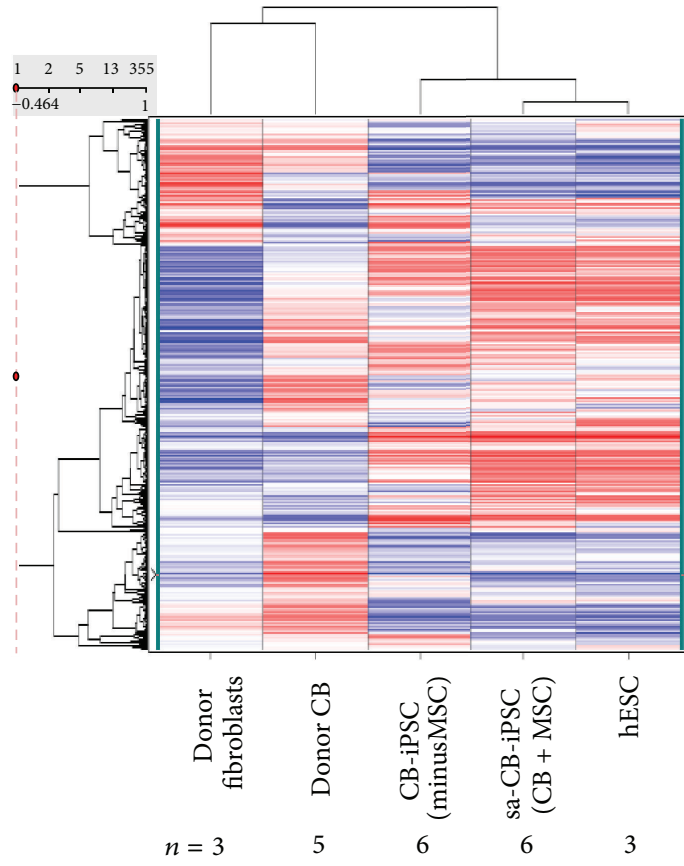

(c)

Figure 5: Continued. 


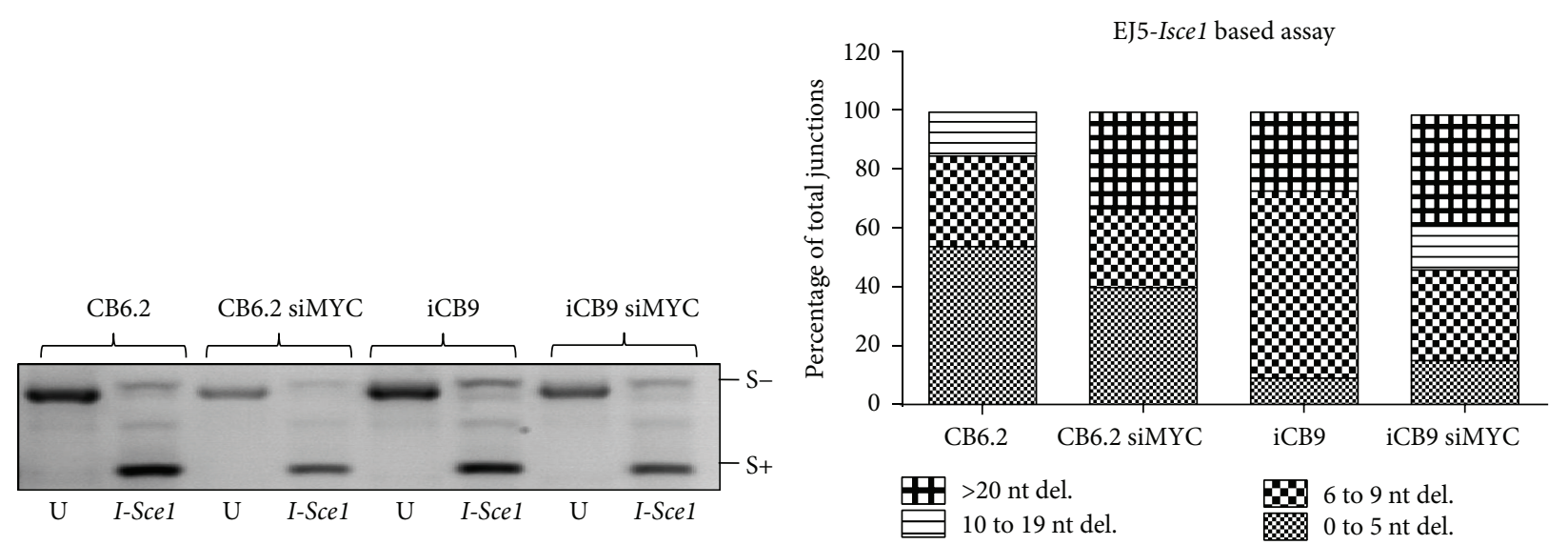

(i)

(ii)

(d)

FIGURE 5: C-MYC maintained high-quality and high-efficiency NHEJ and is required for less error-prone DSB repair. (a)(i) Western blot analysis from whole cell extracts of $\mathrm{H} 9$ treated with either solvent control (DMSO) or MYC inhibitor (10058-F4) for $24 \mathrm{~h}$ at $50 \mu \mathrm{M}$, exposed to IR (1 Gy), and collected at indicated time points. (a)(ii) Densitometry analysis comparing the means from three independent western blots as in ((a)(i)). Statistical significance of the data was determined using 2-way ANOVA with Bonferroni posttests ( $\gamma \mathrm{H} 2 \mathrm{AX}$ is significantly different between DMSO and MYC inhibition at $1 \mathrm{~h}, 2 \mathrm{~h}$, and $4 \mathrm{~h}, p<0.05$; pATM is significantly different at $1 \mathrm{~h}, p<0.05$; Ku 80 is significantly different at $0 \mathrm{~h}$ and $2 \mathrm{~h}, p<0.05)$. ((b)(i) and (b)(ii)) The graph represents (i) efficiency of end-joining repair and (ii) percentage of misrepair in linearized PUC18 (with compatible ends) following incubation with extracts from H9 cells \pm MYC siRNA. Repair efficiency is calculated by counting the total number of colonies (correctly repaired (blue) + incorrectly repaired (white)) from in vitro assays. Statistical significance was determined using paired $t$-test analysis ( $p<0.01$ between data sets H9 versus H9 siMYC). (c) Shown is the heatmap of $\log _{2}$ meansubtracted normalized values of signal intensities from averaged independent biological replicate microarray samples $(n=3-6$ per condition) representing the expression of genes in MYC module in mRNA from donor fibroblasts, donor CB (CD34 ${ }^{+}$population), and $\mathrm{CB}-\mathrm{iPSC}$ lines (i.e., CB.iPS + MSC and CB.iPS (minusMSC)). ((d)(i) and (d)(ii)) (i) Shown is a representative gel image of the PCR products recovered from CB6.2 and iCB9 with or without treatments with siMYC. The PCR products are either redigested with I-Scel or left uncut (U). (S-) products represent the I-Sce1 resistant repair products. These (S-) fragments are cloned into TOP10 competent cells. (ii) The clones, each representing different repair products, were analyzed by sequencing near I-Scel junction. Data represents $\sim 10-15$ clones analyzed in H9, CB6.2, iCB9, and iHuF3. The data is significantly different $\left({ }^{*} p<0.05\right)$ for CB6.2 versus CB6.2 siMYC ( $>20$ nt deletion) and iCB9 versus iCB9 siMYC $(>20 \mathrm{nt}$ deletion). Results are representative of the mean of two independent experiments of each set $\pm \mathrm{SEM} ;{ }^{*} p<0.05,{ }^{* *} p<0.01$, ${ }^{* * *} p<0.001$, and ${ }^{* * * *} p<0.0001$, based on $t$-test analysis.

cocktail significantly reduces the efficiency of reprogramming [40]. Interestingly, sa-CB-iPSCs were characterized by hESC-like MYC-regulated expression module and robustly expressed MYC complex genes [12]. In a different context, C-MYC has also been shown to regulate the transcription of several key DSB repair genes including Ku70 and BRCA1 in somatic cells [26]. Therefore, we questioned whether CMYC contributes to enhanced efficacy and efficiency of repair in hESCs. As a proof of principle, C-MYC was depleted in hESC H9, using chemical inhibitor 10058-F4, which prevents MYC/MAX association and downstream signaling [41]. Following C-MYC inhibition $(50 \mu \mathrm{M}, 24 \mathrm{~h})$, the control and drug-treated cells were exposed to IR (1 Gy) and cells were examined at $0,1,2$, and 4 hours after IR for expression of DDR proteins by immunoblotting. Notably, compared with untreated cells, C-MYC inhibition resulted in increased levels of $\gamma \mathrm{H} 2 \mathrm{AX} 1 \mathrm{~h}$ after IR and persists until $4 \mathrm{~h}$ after IR (Figures 5(a)(i) and 5(a)(ii)). Whereas pATM expression changes after IR in untreated cells are more subtle, C-MYC inhibition results in persistence of pATM that decreases by $4 \mathrm{~h}$. While $\mathrm{Ku} 80$ expression decreases with C-MYC inhibition, it is not significant compared with controls. These data suggest that
C-MYC is involved in the radiation-induced DSB repair response in hESCs, facilitating repair.

We therefore next determined whether MYC inhibition reduced quality and efficiency of DNA end-joining in $\mathrm{H} 9$ cells. Remarkably, siRNA-mediated MYC knockdown (KD) in $\mathrm{H} 9$ resulted in a significant decrease in total NHEJ efficiency of these cells, as measured by counting total number of colonies (blue plus white) in an in vitro PUC18 assay (Figure 5(b)(i)). Moreover, we also observed a significant increase in the proportion of misrepaired colonies from hESC H9 cells treated with MYC siRNA (Figure 5(b)(ii)).

Since MSC activation of CB donors during reprogramming robustly activated MYC complex genes of pluripotency and facilitated high-capacity reprogramming of human MP differentiated from $\mathrm{CD} 34^{+}$cells [12], we sought to evaluate the MYC module expression networks in sa-CB-iPSC versus other hiPSC lines. Interestingly, microarray expression of MYC-regulated circuit genes in sa-CB-iPSC was more hESClike relative to standard CB-iPSC (Figure 5(c)). We next determined whether inhibition of C-MYC affected the quality of end-joining in these categories of hiPSCs. For these experiments, we utilized I-Scel-based assays (Figure S1) and 
measured DSB repair in these cells in vitro (see Section 2). As shown in Figure 5(d)(i), the majority of the GFP genes amplified from the PCR reaction were S+ (I-Scel sensitive), indicating that these extracts mostly produced distal-end joining products that are error-free. However, to determine the character of the errors from plasmid reactivation, PCR products resistant to IScel restriction digestion (S-) were cloned into PCR2.1. Remarkably, similar to C-MYC depletion end-joining results in $\mathrm{H} 9$ (Figure 5(d)(ii)), analysis of DSB repair junctions indicated that the efficacy of DNA endjoining significantly deteriorated and became more errorprone when C-MYC was depleted in sa-CB-iPSC (CB6.2) $\left({ }^{*} p<0.05\right)$ (Figure 5(d)(ii)). Specifically, while none of the 13 clones from WT CB6.2 had deletions of $>20 \mathrm{nt}$, $33 \%$ of clones ( 5 out of 15 ) showed deletions of $>20$ nt when C-MYC was depleted $\left({ }^{*} p<0.05\right)$. Interestingly, in WT iCB9, 20\% of clones had deletions of $>20$ nt nucleotides that further increased following C-MYC KD (38\% versus $27 \%$ in WT) (Figure 5(d)(ii)). These results imply that C-MYC gene expression signature is linked to efficacious NHEJ DSB repair in pluripotent cells. Moreover, these data indicate that expression of C-MYC gene expression circuits in hiPSCs could be an important indicator of not only overall efficiency of reprogramming, but also overall DDR signaling and, in particular, repair of DSBs.

\section{Discussion}

Generating hiPSCs from adult cells represents one of the most exciting developments in regenerative medicine. However, potential clinical applications of hiPSCs are severely hampered by low efficiency of production and suboptimal genomic integrity. One study estimated that $\sim 13 \%$ of hESC and hiPSC cultures demonstrated aberrant aneuploid karyotypes [42]. Comparative genomic analyses have revealed a high frequency of DNA copy-number variations (CNVs) in hiPSCs when compared to either hESCs or somatic cells of origin $[9,43]$. DNA damage and inaccurate "followup" repair mechanisms likely present a significant source of genomic aberrations [44]. For example, reprogramming methods may introduce DNA lesions in the form of lethal DSBs [44]. DSB lesions are introduced by ectopic expression of reprogramming factors and appear to develop irrespective of the reprogramming methodology (i.e., integrative or nonintegrative) $[44,45]$. DSB repair components also play an important role in controlling the efficiency of reprogramming [44, 46-50]. Cells that are impaired in HR genes, such as BRCA1/BRCA2 or NHEJ factor DNA ligase IV (LIG4), show significantly decreased capacity for reprogramming [44, 49]. However, it is not well understood whether the features that promote reprogramming further translate into hiPSCs with more robust and efficacious DSB repair properties.

Our study demonstrates that CB-iPSCs generated with high efficiency (sa-CB-iPSC) possess an hESC-like C-MYC transcript signature and have a DDR that more closely resembles hESCs, relative to hiPSCs derived via standard methods. Moreover, sa-CB-iPSCs also performed end-joining DSB repair with less errors, compared with standard CB.iPSCs. Notably, depletion of C-MYC led to increased end-joining errors, suggesting for the first time that MYC-regulated circuits may be required for maintaining genomic integrity in hiPSCs.

Cell differentiation leads to a decline in DNA repair capacity, which can further lead to accumulation of DNA damage and mutations $[32,33]$. In contrast, stem progenitors possess greater overall capacity for efficient DNA repair. Stem-progenitor cells may also be more amenable to cellular reprogramming, compared with differentiated somatic cells $[12,51,52]$. However, sa-CB-iPSCs derived from human myeloid progenitors through MSC activation signals are generated even more efficiently (1-4\%) and possess minimal interline variability when differentiated to vascular progenitors, compared with hiPSCs derived from CB mononuclear cells generated without MSC activation (0.2-0.3\%) [52]. While no significant differences in baseline expression of mRNA transcripts and translated proteins for DDR genes were observed between CB-iPSCs derived via different methods, most significant differences emerged when these cells were analyzed for their DSB repair activities. sa-CB-iPSCs exhibited end-joining repair which was less error-prone and more closely resembles DSB repair properties in hESCs.

Repair of nonligatable ends by NHEJ requires an endprocessing step for ligation and thus is prone to errors resulting in deletions of a few nucleotides at DSB repair junctions. IR damage induces NHEJ-mediated DNA misrepair events in late G2 cell cycle stage [53]. Interestingly, ATM suppresses genomic aberrations and incorrect end utilization during NHEJ, known as "distal-end joining," formed as a consequence of multiple DSBs due to genotoxic stress $[54,55]$. Although hESCs can uniquely employ high-fidelity NHEJ that can operate independently of ATM [56], hiPSCs perform error-prone DSB repair in particular when exposed to genotoxic stress [53]. Our studies indicate that despite similarities in levels of total ATM and ATM phosphorylation kinetics after IR, sa-CB-iPSCs and standard CB-iPSC have differences in NHEJ responses. In particular, standard fibroblast and CB-iPSCs demonstrated a higher percentage of large deletions ( $\geq 20 \mathrm{nt}$ ) in DSB junctions, compared to sa-CBiPSCs and hESCs. Remarkably, "error-proneness" of NHEJ significantly escalates when pluripotent cells are subjected to IR stress under conditions of MYC inhibition.

MYC is an important regulator of transcription in hESCs and is one of the key factors employed in the generation of hiPSCs. Indeed, ectopic MYC is necessary for efficiently generating iPSCs $[57,58]$. MYC interacts with the NuA4 complex, a regulator of ESC identity. and is the master regulator of a key ESC transcription program [14, 59, 60]. MYC also activates high telomerase activity during reprogramming via regulation of TERT [61]. Hematopoietic growth factor (GF) stimulation of myeloid progenitors differentiated from CD $34^{+}$CB cells activates C-MYC-regulated modules to hESC-like levels and facilitates their pluripotency induction [12]. These GF-activated progenitors robustly overexpress MYC complex genes, which have been found to be vital for pluripotency and facilitation of somatic reprogramming [12]. Interestingly, the C-MYC module signature in ESCs highly resembles the C-MYC module that is found in cancer cells [39]. Our data reveals that hESCs and sa-CB-iPSCs 
have a similar C-MYC module signature. Moreover, MYC inhibition results in more repair errors in hESC and hiPSCs. Thus, while NHEJ in somatic cells is considered error-prone [19], in normal pluripotent cells, C-MYC appears to be required for maintaining a more error-free NHEJ repair. Notably, putative C-MYC binding sites have been identified in the regulatory regions of several NHEJ genes, suggesting a potential mechanism through which C-MYC may maintain error-free NHEJ in hESCs and hiPSCs [26, 62].

In conclusion, our studies show that the various methods for generating hiPSCs may affect the pathways that regulate genomic integrity. Further characterization is required to determine how these pathways are interconnected and will enable improvement of the genomic integrity of hiPSCs. Knowing that C-MYC is also a master regulator of chromatin modifications $[13,60]$, its role in facilitating repair might be not only transcriptionally regulated but also epigenetically controlled. Thus, further elucidation of the role of C-MYC in maintenance of genomic integrity, regulating the balance between "good repair" and "bad repair" in pluripotent cells, is required.

\section{Competing Interests}

Under a licensing agreement between Life Technologies and the JHU, Elias T. Zambidis is entitled to a share of royalty received by the university for licensing of stem cells. The terms of this arrangement are managed by JHU in accordance with its conflict of interests policies. This does not alter the authors' adherence to journal policies on sharing data and materials.

\section{Acknowledgments}

The authors thank Dr. Igor Slukvin (University of WisconsinMadison) for providing the iCB2.5, iCB8, and iCB9 line and Dr. Renee Reijo-Pera (Stanford University) for providing the iHuF3 cell line. This work was supported by grants from the NIH/NHLBI (U01HL099775, Elias T. Zambidis), NIH/NEI (R01EY023962, Elias T. Zambidis), NIH/NICHD (R01HD082098, Elias T. Zambidis), NIH/NCI (T32CA60441, Jeffrey S Huo), and the Maryland Stem Cell Research Fund (2011-MSCRF-II-0008-00, Elias T. Zambidis; 2012-MSCRFIII-033, Jeffrey S. Huo; 2014-MSCRF-II-118153, Tea Soon Park; and 2014-MSCRF-SC-0614105, Feyruz V. Rassool).

\section{References}

[1] C. N. Svendsen, "Back to the future: how human induced pluripotent stem cells will transform regenerative medicine," Human Molecular Genetics, vol. 22, no. 1, Article ID ddt379, pp. R32-R38, 2013.

[2] A. Trounson, K. A. Shepard, and N. D. DeWitt, "Human disease modeling with induced pluripotent stem cells," Current Opinion in Genetics and Development, vol. 22, no. 5, pp. 509-516, 2012.

[3] B. Feng, J.-H. Ng, J.-C. D. Heng, and H.-H. Ng, "Molecules that promote or enhance reprogramming of somatic cells to induced pluripotent stem cells," Cell Stem Cell, vol. 4, no. 4, pp. 301-312, 2009.
[4] K. Tanabe, M. Nakamura, M. Narita, K. Takahashi, and S. Yamanaka, "Maturation, not initiation, is the major roadblock during reprogramming toward pluripotency from human fibroblasts," Proceedings of the National Academy of Sciences of the United States of America, vol. 110, no. 30, pp. 12172-12179, 2013.

[5] I. Wilmut, G. Sullivan, and I. Chambers, "The evolving biology of cell reprogramming," Philosophical Transactions of the Royal Society B: Biological Sciences, vol. 366, no. 1575, pp. 2183-2197, 2011.

[6] Y. Xu, X. Wei, M. Wang et al., "Proliferation rate of somatic cells affects reprogramming efficiency," Journal of Biological Chemistry, vol. 288, no. 14, pp. 9767-9778, 2013.

[7] U. Ben-David, N. Benvenisty, and Y. Mayshar, "Genetic instability in human induced pluripotent stem cells: classification of causes and possible safeguards," Cell Cycle, vol. 9, no. 23, pp. 4603-4604, 2010.

[8] A. Gore, Z. Li, H.-L. Fung et al., "Somatic coding mutations in human induced pluripotent stem cells," Nature, vol. 471, no. 7336, pp. 63-67, 2011.

[9] S. M. Hussein, N. N. Batada, S. Vuoristo et al., "Copy number variation and selection during reprogramming to pluripotency," Nature, vol. 471, no. 7336, pp. 58-62, 2011.

[10] Y. Mayshar, U. Ben-David, N. Lavon et al., "Identification and classification of chromosomal aberrations in human induced pluripotent stem cells," Cell Stem Cell, vol. 7, no. 4, pp. 521-531, 2010.

[11] D. A. Robinton and G. Q. Daley, "The promise of induced pluripotent stem cells in research and therapy," Nature, vol. 481, no. 7381, pp. 295-305, 2012.

[12] T. S. Park, J. S. Huo, A. Peters et al., "Growth factor-activated stem cell circuits and stromal signals cooperatively accelerate non-integrated iPSC reprogramming of human myeloid progenitors," PLoS ONE, vol. 7, no. 8, Article ID e42838, 2012.

[13] R. Sridharan, J. Tchieu, M. J. Mason et al., "Role of the murine reprogramming factors in the induction of pluripotency," Cell, vol. 136, no. 2, pp. 364-377, 2009.

[14] X. Chen, H. Xu, P. Yuan et al., "Integration of external signaling pathways with the core transcriptional network in embryonic stem cells," Cell, vol. 133, no. 6, pp. 1106-1117, 2008.

[15] R. L. Judson, J. E. Babiarz, M. Venere, and R. Blelloch, "Embryonic stem cell-specific microRNAs promote induced pluripotency," Nature Biotechnology, vol. 27, no. 5, pp. 459-461, 2009.

[16] N. Hyka-Nouspikel, J. Desmarais, P. J. Gokhale et al., "Deficient DNA damage response and cell cycle checkpoints lead to accumulation of point mutations in human embryonic stem cells," STEM CELLS, vol. 30, no. 9, pp. 1901-1910, 2012.

[17] P. Nagaria, C. Robert, and F. V. Rassool, "DNA double-strand break response in stem cells: mechanisms to maintain genomic integrity," Biochimica et Biophysica Acta (BBA)_General Subjects, vol. 1830, no. 2, pp. 2345-2353, 2013.

[18] T. Helleday, "Pathways for mitotic homologous recombination in mammalian cells," Mutation Research/Fundamental and Molecular Mechanisms of Mutagenesis, vol. 532, no. 1-2, pp. 103115, 2003.

[19] F. V. Rassool and A. E. Tomkinson, “Targeting abnormal DNA double strand break repair in cancer," Cellular and Molecular Life Sciences, vol. 67, no. 21, pp. 3699-3710, 2010.

[20] J. Fan, C. Robert, Y.-Y. Jang et al., "Human induced pluripotent cells resemble embryonic stem cells demonstrating enhanced levels of DNA repair and efficacy of nonhomologous endjoining," Mutation Research, vol. 713, no. 1-2, pp. 8-17, 2011. 
[21] L. Serrano, L. Liang, Y. Chang et al., "Homologous recombination conserves DNA sequence integrity throughout the cell cycle in embryonic stem cells," Stem Cells and Development, vol. 20, no. 2, pp. 363-374, 2011.

[22] L. Z. Luo, S. Gopalakrishna-Pillai, S. L. Nay et al., "DNA repair in human pluripotent stem cells is distinct from that in nonpluripotent human cells," PLoS ONE, vol. 7, no. 3, article e30541, 2012.

[23] V. Ramos-Mejia, M. Mũoz-Lopez, J. L. Garcia-Perez, and P. Menendez, "iPSC lines that do not silence the expression of the ectopic reprogramming factors may display enhanced propensity to genomic instability," Cell Research, vol. 20, no. 10, pp. 1092-1095, 2010.

[24] F. J. Molina-Estevez, M. L. Lozano, S. Navarro et al., "Brief report: impaired cell reprogramming in nonhomologous end joining deficient cells," Stem Cells, vol. 31, no. 8, pp. 1726-1730, 2013.

[25] C. Y. Lin, J. Lovén, P. B. Rahl et al., "Transcriptional amplification in tumor cells with elevated c-Myc," Cell, vol. 151, no. 1, pp. 56-67, 2012.

[26] K. R. Luoto, A. X. Meng, A. R. Wasylishen et al., "Tumor cell kill by c-MYC depletion: role of MYC-regulated genes that control DNA double-strand break repair," Cancer Research, vol. 70, no. 21, pp. 8748-8759, 2010.

[27] J. A. Byrne, H. N. Nguyen, and R. A. Reijo Pera, "Enhanced generation of induced pluripotent stem cells from a subpopulation of human fibroblasts," PLoS ONE, vol. 4, no. 9, article e7118, 2009.

[28] P. W. Burridge, S. Thompson, M. A. Millrod et al., "A universal system for highly efficient cardiac differentiation of human induced pluripotent stem cells that eliminates interline variability," PLoS ONE, vol. 6, no. 4, Article ID e18293, 2011.

[29] K. Hu, J. Yu, K. Suknuntha et al., "Efficient generation of transgene-free induced pluripotent stem cells from normal and neoplastic bone marrow and cord blood mononuclear cells," Blood, vol. 117, no. 14, pp. e109-e119, 2011.

[30] L. A. Tobin, C. Robert, P. Nagaria et al., "Targeting abnormal DNA repair in therapy-resistant breast cancers," Molecular Cancer Research, vol. 10, no. 1, pp. 96-107, 2012.

[31] N. Bennardo, A. Cheng, N. Huang, and J. M. Stark, "AlternativeNHEJ is a mechanistically distinct pathway of mammalian chromosome break repair," PLoS Genetics, vol. 4, no. 6, Article ID e1000110, 2008.

[32] J.-H. Chen, C. N. Hales, and S. E. Ozanne, "DNA damage, cellular senescence and organismal ageing: causal or correlative?" Nucleic Acids Research, vol. 35, no. 22, pp. 7417-7428, 2007.

[33] A. Seluanov, D. Mittelman, O. M. Pereira-Smith, J. H. Wilson, and V. Gorbunova, "DNA end joining becomes less efficient and more error-prone during cellular senescence," Proceedings of the National Academy of Sciences of the United States of America, vol. 101, no. 20, pp. 7624-7629, 2004.

[34] O. Momcilovic, L. Knobloch, J. Fornsaglio, S. Varum, C. Easley, and G. Schatten, "DNA damage responses in human induced pluripotent stem cells and embryonic stem cells," PLoS ONE, vol. 5, no. 10, Article ID e13410, 2010.

[35] C. Thiriet and J. J. Hayes, "Chromatin in need of a fix: phosphorylation of H2AX connects chromatin to DNA repair," Molecular Cell, vol. 18, no. 6, pp. 617-622, 2005.

[36] Y. Xu and D. Baltimore, "Dual roles of ATM in the cellular response to radiation and in cell growth control," Genes and Development, vol. 10, no. 19, pp. 2401-2410, 1996.
[37] M. Ashcroft, M. H. G. Kubbutat, and K. H. Vousden, "Regulation of p53 function and stability by phosphorylation," Molecular and Cellular Biology, vol. 19, no. 3, pp. 1751-1758, 1999.

[38] A. Gunn and J. M. Stark, "I-SceI-based assays to examine distinct repair outcomes of mammalian chromosomal double strand breaks," Methods in Molecular Biology, vol. 920, pp. 379391, 2012.

[39] J. Kim, A. J. Woo, J. Chu et al., "A Myc network accounts for similarities between embryonic stem and cancer cell transcription programs," Cell, vol. 143, no. 2, pp. 313-324, 2010.

[40] M. Nakagawa, M. Koyanagi, K. Tanabe et al., "Generation of induced pluripotent stem cells without Myc from mouse and human fibroblasts," Nature Biotechnology, vol. 26, no. 1, pp. 101106, 2008.

[41] H. Wang, D. I. Hammoudeh, A. V. Follis et al., "Improved low molecular weight Myc-Max inhibitors," Molecular Cancer Therapeutics, vol. 6, no. 9, pp. 2399-2408, 2007.

[42] S. M. Taapken, B. S. Nisler, M. A. Newton et al., "Karotypic abnormalities in human induced pluripotent stem cells and embryonic stem cells," Nature Biotechnology, vol. 29, no. 4, pp. 313-314, 2011.

[43] L. C. Laurent, I. Ulitsky, I. Slavin et al., "Dynamic changes in the copy number of pluripotency and cell proliferation genes in human ESCs and iPSCs during reprogramming and time in culture," Cell Stem Cell, vol. 8, no. 1, pp. 106-118, 2011.

[44] F. González, D. Georgieva, F. Vanoli et al., "Homologous recombination DNA repair genes play a critical role in reprogramming to a pluripotent state," Cell Reports, vol. 3, no. 3, pp. 651-660, 2013.

[45] T. Kawamura, J. Suzuki, Y. V. Wang et al., "Linking the p53 tumour suppressor pathway to somatic cell reprogramming," Nature, vol. 460, no. 7259, pp. 1140-1144, 2009.

[46] S.-H. Chiou, B.-H. Jiang, Y.-L. Yu et al., "Poly(ADP-ribose) polymerase 1 regulates nuclear reprogramming and promotes iPSC generation without c-Myc," Journal of Experimental Medicine, vol. 210, no. 1, pp. 85-98, 2013.

[47] T. Kinoshita, G. Nagamatsu, T. Kosaka et al., "Ataxiatelangiectasia mutated (ATM) deficiency decreases reprogramming efficiency and leads to genomic instability in iPS cells," Biochemical and Biophysical Research Communications, vol. 407, no. 2, pp. 321-326, 2011.

[48] F. J. Molina-Estevez, M. L. Lozano, S. Navarro et al., "Impaired cell reprogramming in non-homologous end joining deficient cells," STEM CELLS, vol. 31, no. 8, pp. 1726-1730, 2013.

[49] K. Tilgner, I. Neganova, I. Moreno-Gimeno et al., "A human iPSC model of Ligase IV deficiency reveals an important role for NHEJ-mediated-DSB repair in the survival and genomic stability of induced pluripotent stem cells and emerging haematopoietic progenitors," Cell Death and Differentiation, vol. 20, no. 8, pp. 1089-1100, 2013.

[50] S. K. Yung, K. Tilgner, M. H. Ledran et al., "Brief report: human pluripotent stem cell models of fanconi anemia deficiency reveal an important role for fanconi anemia proteins in cellular reprogramming and survival of hematopoietic progenitors," STEM CELLS, vol. 31, no. 5, pp. 1022-1029, 2013.

[51] J. B. Kim, H. Zaehres, G. Wu et al., "Pluripotent stem cells induced from adult neural stem cells by reprogramming with two factors," Nature, vol. 454, no. 7204, pp. 646-650, 2008.

[52] T. S. Park, I. Bhutto, L. Zimmerlin et al., "Vascular progenitors from cord blood-derived induced pluripotent stem cells possess augmented capacity for regenerating ischemic retinal vasculature," Circulation, vol. 129, no. 3, pp. 359-372, 2014. 
[53] A. N. Bogomazova, M. A. Lagarkova, L. V. Tskhovrebova, M. V. Shutova, and S. L. Kiselev, "Error-prone nonhomologous end joining repair operates in human pluripotent stem cells during late G2," Aging, vol. 3, no. 6, pp. 584-596, 2011.

[54] N. Bennardo and J. M. Stark, "ATM limits incorrect end utilization during non- homologous end joining of multiple chromosome breaks," PLoS Genetics, vol. 6, no. 11, article e1001194, 2010.

[55] A. Gunn, N. Bennardo, A. Cheng, and J. M. Stark, "Correct end use during end joining of multiple chromosomal double strand breaks is influenced by repair protein RAD50, DNA-dependent protein kinase DNA-PKcs, and transcription context," The Journal of Biological Chemistry, vol. 286, no. 49, pp. 4247042482, 2011.

[56] B. R. Adams, A. J. Hawkins, L. F. Povirk, and K. Valerie, "ATM-independent, high-fidelity nonhomologous end joining predominates in human embryonic stem cells," Aging, vol. 2, no. 9, pp. 582-596, 2010.

[57] G. Nagamatsu, S. Saito, T. Kosaka et al., "Optimal ratio of transcription factors for somatic cell reprogramming," Journal of Biological Chemistry, vol. 287, no. 43, pp. 36273-36282, 2012.

[58] N. V. Varlakhanova, R. F. Cotterman, W. N. deVries et al., "Myc maintains embryonic stem cell pluripotency and self-renewal," Differentiation, vol. 80, no. 1, pp. 9-19, 2010.

[59] L. A. Boyer, T. I. Lee, M. F. Cole et al., "Core transcriptional regulatory circuitry in human embryonic stem cells," Cell, vol. 122, no. 6, pp. 947-956, 2005.

[60] J. Kim, J. Chu, X. Shen, J. Wang, and S. H. Orkin, "An extended transcriptional network for pluripotency of embryonic stem cells," Cell, vol. 132, no. 6, pp. 1049-1061, 2008.

[61] R. M. Marion, K. Strati, H. Li et al., "Telomeres acquire embryonic stem cell characteristics in induced pluripotent stem cells," Cell Stem Cell, vol. 4, no. 2, pp. 141-154, 2009.

[62] N. Muvarak, S. Kelley, C. Robert et al., "c-MYC generates repair errors via increased transcription of alternative-NHEJ factors, LIG3 and PARP1, in tyrosine kinase-activated leukemias," Molecular Cancer Research, vol. 13, no. 4, pp. 699-712, 2015. 

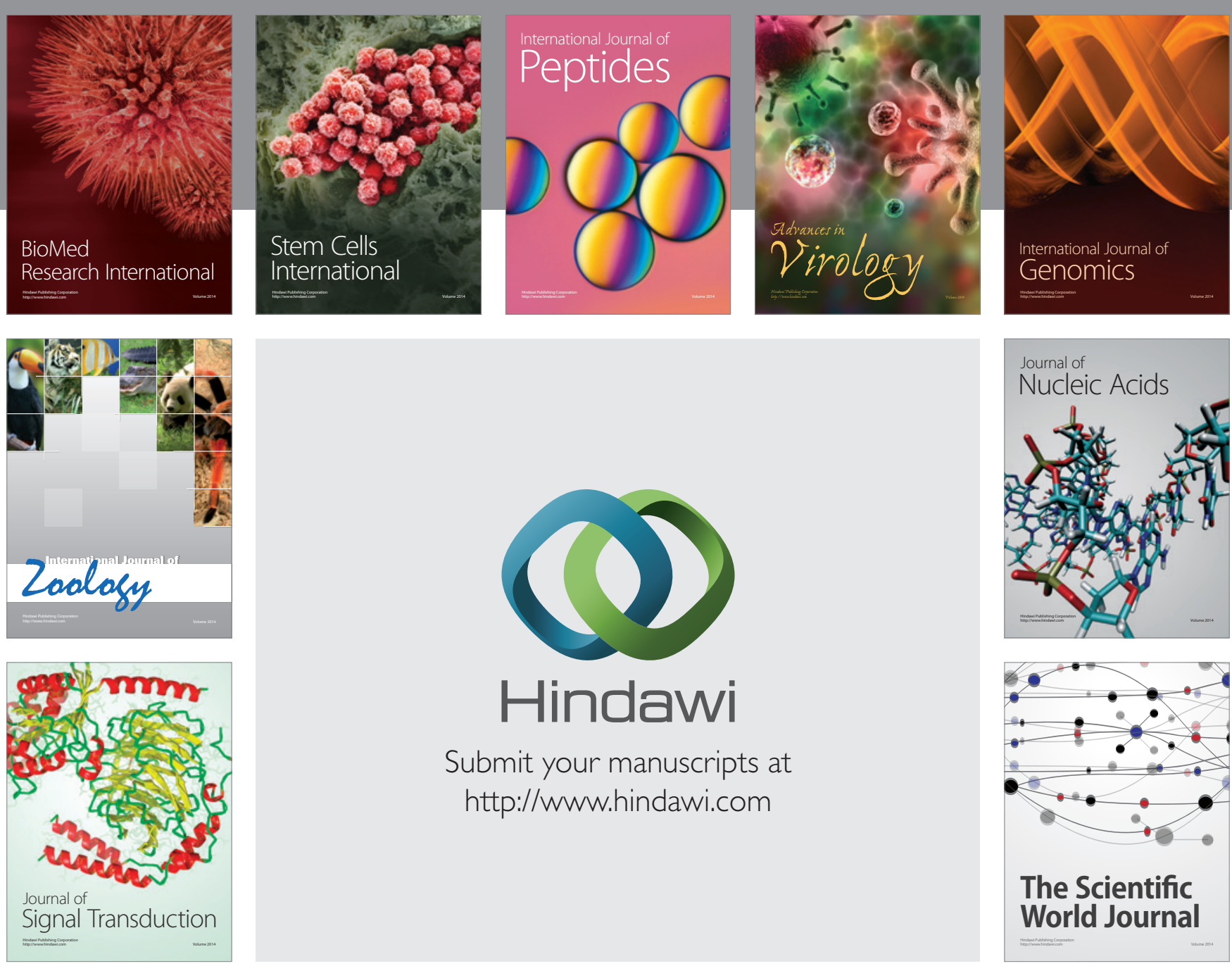

Submit your manuscripts at

http://www.hindawi.com
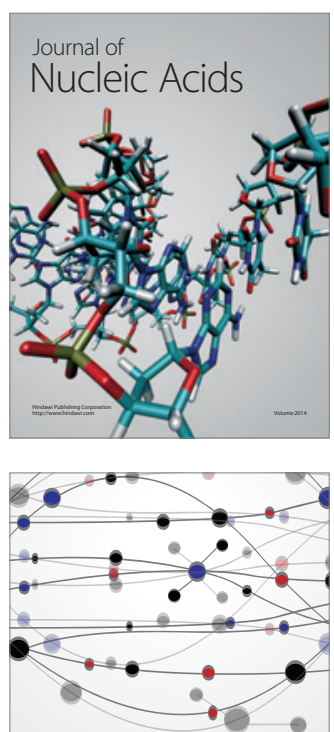

The Scientific World Journal
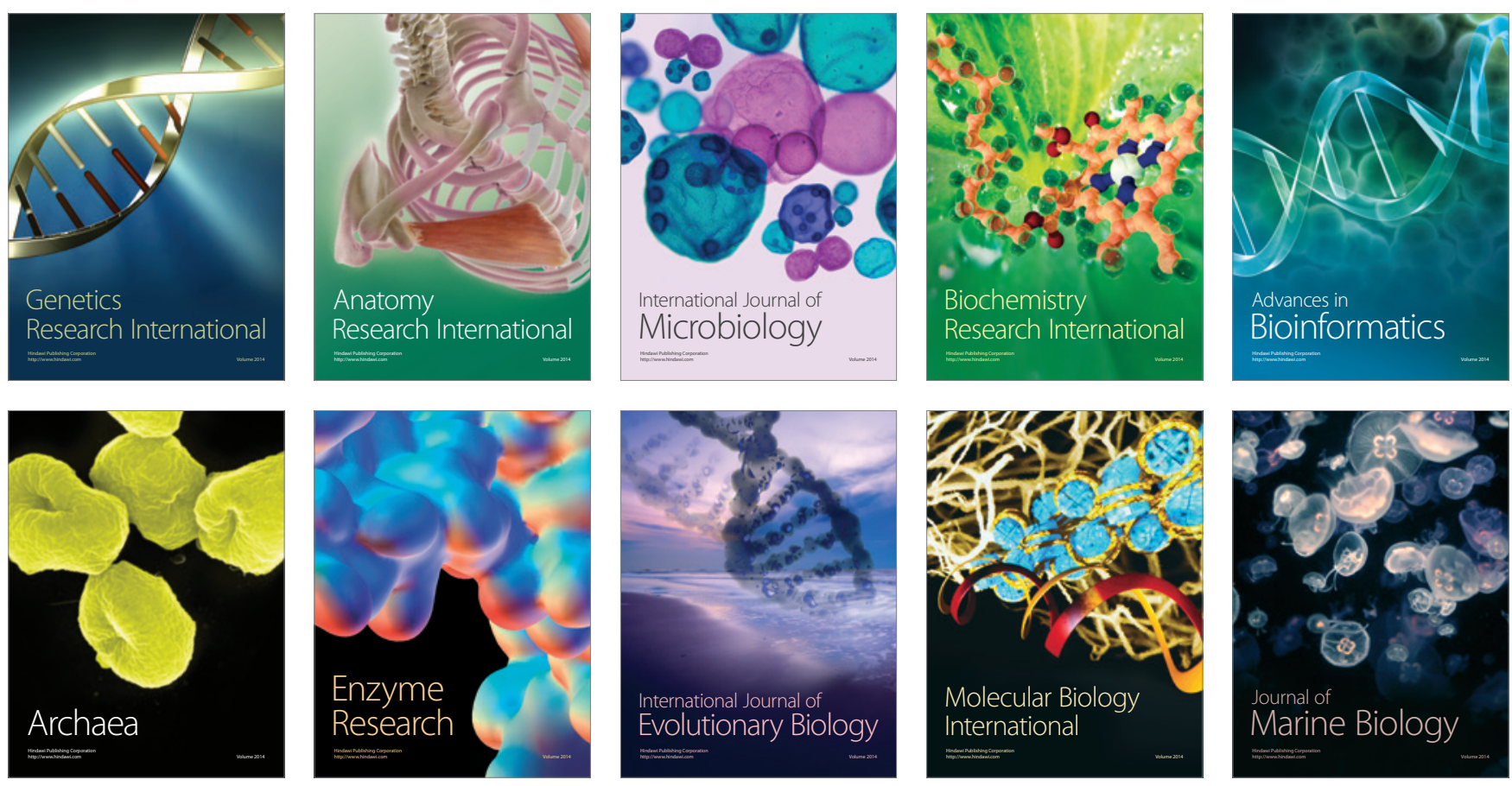